\title{
Comprehensive analysis of the Corynebacterium glutamicum transcriptome using an improved RNAseq technique
}

\author{
Katharina Pfeifer-Sancar ${ }^{1}$, Almut Mentz ${ }^{1}$, Christian Rückert ${ }^{1,2}$ and Jörn Kalinowski 1,2*
}

\begin{abstract}
Background: The use of RNAseq to resolve the transcriptional organization of an organism was established in recent years and also showed the complexity and dynamics of bacterial transcriptomes. The aim of this study was to comprehensively investigate the transcriptome of the industrially relevant amino acid producer and model organism Corynebacterium glutamicum by RNAseq in order to improve its genome annotation and to describe important features for transcription and translation.
\end{abstract}

Results: RNAseq data sets were obtained by two methods, one that focuses on $5^{\prime}$-ends of primary transcripts and another that provides the overall transcriptome with an improved resolution of $3^{\prime}$-ends of transcripts. Subsequent data analysis led to the identification of more than 2,000 transcription start sites (TSSs), the definition of 5'-UTRs (untranslated regions) for annotated protein-coding genes, operon structures and many novel transcripts located between or in antisense orientation to protein-coding regions. Interestingly, a high number of mRNAs (33\%) is transcribed as leaderless transcripts. From the data, consensus promoter and ribosome binding site (RBS) motifs were identified and it was shown that the majority of genes in C. glutamicum are transcribed monocistronically, but operons containing up to 16 genes are also present.

Conclusions: The comprehensive transcriptome map of C. glutamicum established in this study represents a major step forward towards a complete definition of genetic elements (e.g. promoter regions, gene starts and stops, 5'-UTRs, RBSs, transcript starts and ends) and provides the ideal basis for further analyses on transcriptional regulatory networks in this organism. The methods developed are easily applicable for other bacteria and have the potential to be used also for quantification of transcriptomes, replacing microarrays in the near future.

Keywords: Corynebacterium glutamicum, RNA, High-throughput sequencing, Transcriptome

\section{Background}

Corynebacterium glutamicum is a non-pathogenic, nonsporulating, gram-positive soil bacterium that belongs to the order Actinomycetales. This microorganism is widely used for the production of various amino acids and other industrially relevant compounds $[1,2]$. Furthermore, the availability of genetic engineering methods, an easy cultivation and a generally-regarded-as-safe status has helped to make it a model organism for systems biology investigations in the Corynebacterineae, comprising important

\footnotetext{
* Correspondence: joern.kalinowski@cebitec.uni-bielefeld.de

${ }^{1}$ Microbial Genomics and Biotechnology, Center for Biotechnology, Bielefeld University, Universitätsstraße 27, 33615, Bielefeld, Germany

${ }^{2}$ Technology Platform Genomics, Center for Biotechnology, Bielefeld

University, Universitätsstraße 27, 33615, Bielefeld, Germany
}

pathogens such as Corynebacterium diphtheriae and Mycobacterium tuberculosis [3]. The genome sequence of the $3.3 \mathrm{Mb}$ circular chromosome was established a decade ago [4,5] and contains more than 3,000 annotated proteincoding sequences (CDS). Based on the complete genome sequence, transcriptional regulation in C. glutamicum has been studied extensively [6] and revealed a complex regulatory network including 97 transcriptional regulator proteins with so far 1,432 regulatory interactions [7]. In addition, C. glutamicum possesses seven sigma factors regulating transcription on a global scale, recognizing specific promoter signals [8]. Although the promoters of about 200 genes have been identified in the last two decades, this

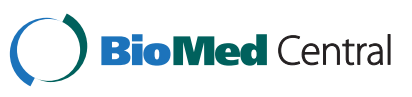

(c) 2013 Pfeifer-Sancar et al.; licensee BioMed Central Ltd. This is an open access article distributed under the terms of the Creative Commons Attribution License (http://creativecommons.org/licenses/by/2.0), which permits unrestricted use, distribution, and reproduction in any medium, provided the original work is properly cited. 
leaves the majority of genes without known transcription signals. Recently, small RNAs (sRNA) were studied in C. glutamicum on a global scale, demonstrating the usefulness of the recently developed method of high-throughput sequencing of cDNA (RNAseq) [9].

The understanding and deciphering of transcriptome complexity of an organism and the underlying functionalities have become a major focus for post-genome research in recent years $[10,11]$. Beside the classical approaches for profiling transcripts like Northern blots, reverse-transcriptase (q)PCR, RACE (rapid amplification of cDNA ends), and microarrays, the recent development of RNAseq has revolutionized transcriptomics. This allows to analyze transcriptomes not only in a completely comprehensive way but also with singlenucleotide resolution $[12,13]$. The features of RNAseq that are unmatched by the classical approaches, i.e. no background or saturation effects as in fluorescence-based detection, no cross-hybridization, and therefore an almost unrestricted dynamic range of detection, make RNAseq an attractive approach to analyze the entire transcriptome also quantitatively [12].

This novel sequencing approach has been successfully applied for studying whole-genome transcription for various prokaryotes and eukaryotes [14-20] and revealed an unexpected complexity of these transcriptomes, e.g. widespread antisense transcription and an enormous amount of small and novel RNAs in bacterial genomes [12,21-24]. Additionally, this method offers the opportunity to improve genome annotation for prokaryotes and eukaryotes, in the latter especially regarding exon identification and alternative splicing effects $[12,25,26]$.

Beside the use of RNAseq results mapping of complete transcripts, detection of transcription start sites and the analysis of the respective promoters, the transcriptome data can be further analyzed to characterize RBSs, providing important information also on translational processes.

Quite often 5'-UTRs have sizes up to several hundred bases, indicating more complex transcriptional and translational functions such as riboswitches, RNA thermometers or binding sites for regulatory RNAs [14,15,21,22]. In contrast, RNAseq analyses have demonstrated that some transcripts are leaderless, imposing a different translation mechanism [14,15].

Further important information obtainable from RNAseq data is the arrangement of genes in operons. The classical operon has multiple genes which are transcribed from a single promoter $[27,28]$. Operons typically contain genes that are functionally connected, e.g. in a metabolic pathway $[29,30]$. Hence, this feature might be helpful in prediction of gene function. Moreover, recent RNAseq data showed that various operons have to be divided into sub-operons due to internal transcription start sites which often respond to different conditions $[15,21]$. Thus, operon structures are not always simple, but can have rather complex architectures that can be fully resolved by RNAseq analysis.

The RNAseq workflow is complex and a number of technical obstacles have to be overcome. First, the majority of total RNA in a bacterial cell consists of ribosomal RNA (> 95\% rRNA). This rRNA has to be removed efficiently either by hybridization-based rRNA depletion or enzyme-based degradation of processed transcripts (including rRNA) [22]. Second, it is important to maintain the strand information to be able to discriminate between sense and antisense transcripts. Methods allowing to obtain this information have been developed for eukaryotes [19,31], and were also adapted for prokaryotes $[15,32]$.

For bacterial RNAseq studies Illumina, 454 and SOLiD sequencing platforms have been used [14,15,17,26,33]. For RNAseq analyses of organisms with known genome sequence, a high number of short reads $(20-50 \mathrm{nt})$ is preferable to a small number of long reads, at least for microbial genomes.

In this study we describe an improved RNAseq method that provides a strand-specific characterization of entire transcriptomes at a whole genome level using high-throughput sequencing. Furthermore, we developed two RNAseq library preparation protocols that allow for analyzing the primary transcriptome and the total transcriptome of an organism separately. Additionally, we applied these two RNAseq protocols successfully to the transcriptome of C. glutamicum. By sequencing the primary transcriptome, we utilized RNA samples from exponential growth phase to analyze TSSs recognized by the housekeeping sigma factor $\sigma^{\mathrm{A}}$. The investigation of the obtained RNAseq data delivered more than 2,000 TSSs which helped to correct more than 200 gene starts and the detection of a quite high number of leaderless transcripts (> 700). For sequencing the whole transcriptome we used RNA samples from nine different conditions (exponential growth phase, heat and cold shock, salt stress, oxidative stresses, and ethanol stress) to obtain a broad range of transcripts. The analysis of this data leads to the identification of operon structures and the detection of novel transcripts in C. glutamicum.

\section{Results}

\section{Development of native 5 '-end and whole transcript RNAseq protocols}

To analyze the whole transcriptome as well as the native transcription start sites of C. glutamicum, i.e. those that originate from initiation of transcription by RNA polymerase, a whole transcriptome RNAseq protocol (Figure 1a) and a native 5'-end RNAseq protocol were developed (Figure 1b), adapting the differential RNA-seq approach [15]. Key differences of our protocol compared to the differential RNA-seq method include: 


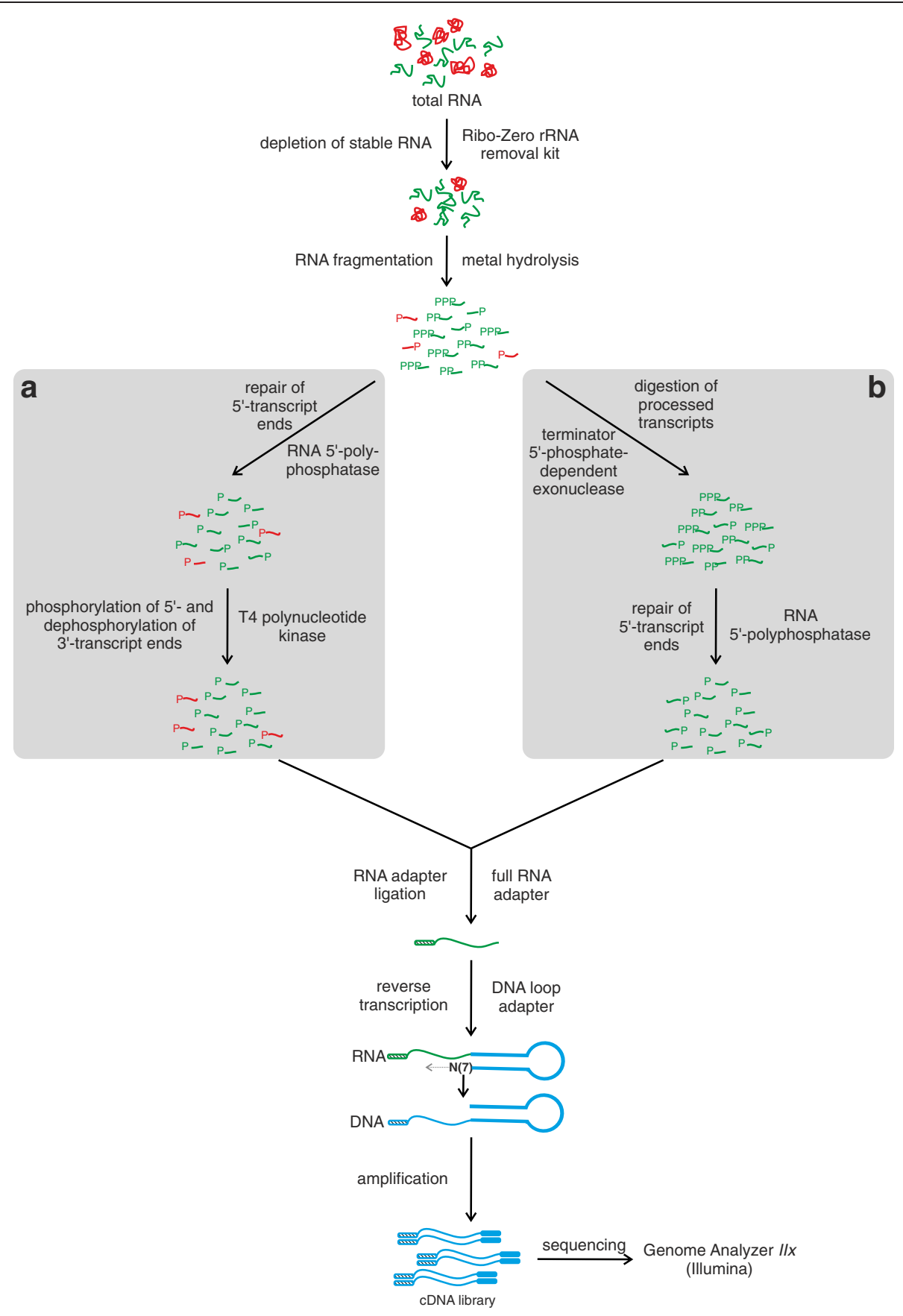

Figure 1 Experimental workflow for the preparation of a whole transcriptome library (a) and of a library enriched for primary

$\mathbf{5}^{\prime}$-transcript ends (b). Both protocols start with isolated total RNA. Stable RNA is then depleted using the Ribo-Zero rRNA removal kit and the obtained RNA is fragmented my metal hydrolysis to a size of 200 - $500 \mathrm{nt}$. For the whole transcriptome library (a) the $5^{\prime}$-triphosphate ends are processed to 5'-monophosphate ends by a RNA 5'-polyphosphatase, unphosphorylated 5'-ends are phosphorylated, and phosphorylated 3'-ends are then dephosphorylated using T4 polynucleotide kinase. For the native $5^{\prime}$-end protocol (b), all fragments containing a $5^{\prime}$-monophosphate are degraded by treatment with a $5^{\prime}$-phosphate dependent exonuclease and the $5^{\prime}$-triphosphate ends of native transcripts are then processed to 5'-monophosphate ends by a RNA 5'-polyphosphatase. Next, for both libraries RNA adapters are ligated to the 5'-ends carrying a

$5^{\prime}$-monophosphate group. The tagging of the $3^{\prime}$-end of the RNA with flanking sequences necessary for reverse transcription is performed in a ligation-free approach with a loop DNA adapter containing seven unpaired wobble bases at its $3^{\prime}$-end. After reverse transcription of the RNA fragments into CDNA fragments, the CDNA fragments are amplified, tagged with sequencing linkers at their ends by PCR and finally sequenced. Stable RNA species (rRNA, tRNA) are depicted in red, other RNAs are given in green, and DNA in blue. 
I) Depletion of stable RNAs using the Ribo-Zero rRNA removal kit, as preliminary tests indicated that rRNA constitutes up to $99 \%$ of the total RNA in C. glutamicum (data not shown), necessitating an efficient removal in both libraries.

II) RNA fragmentation by metal hydrolysis to allow for more efficient ligation of the 5 '-end RNA adapter and better accessibility for the RNA loop adapter. This should result in an increase of completely reverse transcribed cDNAs carrying both adapter sequences needed for high-throughput sequencing. In case of the native 5 -end protocol, this also causes only fragments containing either a $5^{\prime}$-triphosphate (start of a native transcript) or an unphosphorylated $5^{\prime}$-end (resulting from metal hydrolysis) to be retained upon Terminator $5^{\prime}$-phosphatedependent exonuclease.

III) Repair of the $5^{\prime}$-ends of the RNA fragments to enable ligation of the 5 -end RNA adapter. Both protocols apply an RNA 5'-polyphosphatase treatment converting the $5^{\prime}$-triphosphates of primary transcript ends to monophosphates to allow ligation. Thus in case of the native $5^{\prime}$-end protocol, only fragments derived from a $5^{\prime}$-end of a primary transcript will retain a $5^{\prime}$-monophosphate. In case of the whole transcriptome protocol, an additional treatment with $\mathrm{T} 4$ polynucleotide kinase is performed, phosphorylating all $5^{\prime}$-ends lacking a phosphate group and thus enhancing adapter ligation efficiency.

IV) For cDNA synthesis, a loop adapter is used which hybridizes via a 3 '-NNNNNNN-tail preferentially to the $3^{\prime}$-end of the RNA fragments as the binding there is stabilized by the stacking energy of the formed DNA-RNA duplex. This approach saves a preparation step for the reverse transcription (e.g., polyA-tailing, ligation of a 3 '-adapter) and allows the direct accessibility of the $3^{\prime}$-fragment ends as the sequence used in the stem of the loop adapter is identical to the Illumina Paired-Read Primer 2.

V) Several size selection steps are performed to remove adapter dimers.

VI) Sequencing is done using Illumina technology, allowing for a much deeper sequencing and the possibility to obtain paired-end information

The whole transcriptome RNAseq protocol was developed to determine operon structures, so far unknown transcripts as well as transcript ends. During this procedure the primary transcripts are not enriched, enabling the sequencing of processed and native transcripts. Moreover, the whole transcriptome protocol enables read coverage of entire transcripts, including their 3 '-ends.
As this approach should deliver expression of as many transcripts as possible, the RNA samples for this data set originated from mixed C. glutamicum cell samples grown under different cultivation conditions (minimal or complex media w/o stress application, and minimal medium but stressed with $10 \%$ ethanol, $10 \%$ sodium chloride, $90 \%$ dissolved oxygen, $1 \%$ hydrogen peroxide, $2 \mathrm{mM}$ diamide, heat stress at $50^{\circ} \mathrm{C}$ as well as cold stress at $4^{\circ} \mathrm{C}$ ).

The objective of generating the native $5^{\prime}$-end data set was to identify as many TSSs as possible and to localize promoters, RBSs and $5^{\prime}$-UTRs of C. glutamicum in a comprehensive way. We were mainly interested in TSSs recognized by the housekeeping sigma factor $\sigma^{\mathrm{A}}$. Therefore the RNA samples for this data set originated from mixed C. glutamicum cell samples grown in minimal and in complex media. The mixing of cells from both conditions should ensure transcription of most housekeeping genes, including those involved in anabolism and in catabolism.

\section{Data generation by Illumina sequencing and mapping of DNA sequence reads to the Corynebacterium glutamicum ATCC13032 genome}

In order to characterize the primary and the whole transcriptome of C. glutamicum using RNAseq, the two described RNAseq libraries were sequenced on a Genome Analyzer IIx (Illumina). A total of $2 \times 20.53$ and 20.76 million reads were generated from the whole and the primary transcriptome library, respectively. These reads of 26 nt in length (one low quality base trimmed from the 3 '-end of the sequenced 27 nt reads) were mapped to the C. glutamicum genome. After mapping of reads and removal of duplicate mappings (i.e. reads mapping to repeat regions like rRNAs), 15.96 and 2.65 million reads were mapped uniquely to the genome from the whole and the primary transcriptome library, respectively (Table 1).

\section{Comprehensive identification of transcription start sites from the native 5 '-end data set}

The analysis of the native $5^{\prime}$-end data set resulted in the identification of 3,163 TSSs. This number also contains alternative TSSs that originate from one promoter and such that belong to rRNA and tRNA genes. The total number of TSSs was reduced by merging alternative TSSs (81) and removing TSSs that belong to rRNA and tRNA genes (202) or false-positive TSSs (289). An alternative transcription start was assumed to be in a range of $\leq 1$ base. The +1 position with the highest number of read starts within this range was selected as transcription start. After the elimination of alternative, redundant rRNA and tRNA, and false-positive signals, 2,591 TSSs remain (Figure 2; Additional file 1: Table S1). 
Table 1 Summary of sequencing and mapping statistics for the whole transcriptome and primary 5'-transcript ends library

\begin{tabular}{|c|c|c|}
\hline & $\begin{array}{l}\text { whole } \\
\text { transcriptome }^{a}\end{array}$ & $\begin{array}{l}\text { primary } 5^{\prime}- \\
\text { transcript ends }{ }^{a}\end{array}$ \\
\hline total reads & $2 \times 20.53$ & 20.76 \\
\hline total mappings ${ }^{b}$ & 22.19 & 5.07 \\
\hline total mappings of $\mathrm{CPR}^{\mathrm{C}}$ & 13.91 & - \\
\hline $\begin{array}{l}\text { reads mapping at multiple } \\
\text { positions }^{b}\end{array}$ & 1.19 & 0.68 \\
\hline $\begin{array}{l}\text { CPR mapping at multiple } \\
\text { positions }\end{array}$ & 0.72 & - \\
\hline $\begin{array}{l}\text { reads mapping at a single } \\
\text { position }^{b}\end{array}$ & 15.96 & 2.65 \\
\hline $\begin{array}{l}\text { CPR mapping at a single } \\
\text { position }\end{array}$ & 10.20 & - \\
\hline
\end{tabular}

${ }^{\mathrm{a}}$ in million.

${ }^{b}$ in case of the whole transcriptome data: sum of combined read pairs, forward reads without reverse mates, and reverse reads without forward mates that map to the genome sequence of $C$. glutamicum; in case of primary 5 '-transcript ends data: forward reads that map to the genome sequence of C. glutamicum

${ }^{c} \mathrm{CPR}$ : combined pair of reads.

These TSSs can be classified into two groups: TSSs which belong to annotated genes and TSSs that are assigned to novel transcripts. TSSs belonging to annotated genes were further categorized into 1,264 single TSSs (a single TSS per gene) and 890 multiple TSSs (more than one TSS per gene), the latter occurring at 365 genes. TSS numbers per gene could be as high as six, e.g. as found for cmt1, which encodes a trehalose corynomycolyl transferase (Additional file 2: Figure S1).
TSSs belonging to novel transcripts were classified into three categories: antisense TSSs - the respective transcript is allocated in antisense orientation to an annotated gene; intragenic TSSs - these TSSs are located within annotated genes in sense orientation; intergenic TSSs - these TSSs are located between annotated genes (Figure 2).

In total, 2,154 TSS were assigned to annotated genes, whereby 2,147 of 2,154 belong to genes encoding proteins and the remaining seven were assigned to noncoding RNA (one TSS belonging to 4.5S RNA, M1 RNA, and 6C RNA genes, respectively, as well as four TSSs belonging to tmRNA). In summary, for 1,629 of the 3,043 (53.5\%) actually annotated genes in C. glutamicum [34] a TSS was found. Additionally, 437 TSSs could not be assigned to annotated genes, but to novel, so far unknown transcripts. Overall, 233 of these 437 TSSs belong to antisense transcripts, 186 relate to intragenic transcripts and 18 are assigned to new intergenic transcripts (Figure 2).

\section{Identification of $\sigma^{A}$-dependent promoters}

Promoter motifs are sites on the DNA to which the RNA polymerase attaches in order to start transcription $[35,36]$. In bacteria, different species of sigma factors have been identified that are components of the RNA polymerase holoenzyme, each recognizing a different promoter motif and thus contributing to transcription of a particular set of genes [37-39]. The major sigma factor, $\sigma^{70}$, has been found in all known bacterial species and is responsible for the transcription of housekeeping

a

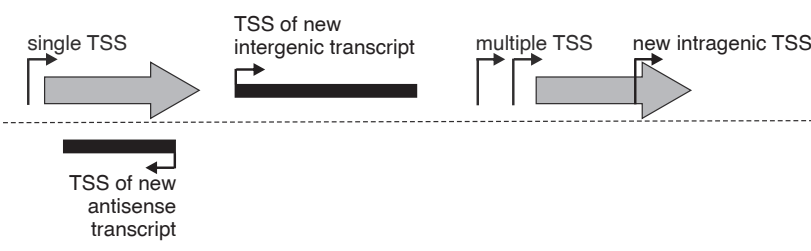

b

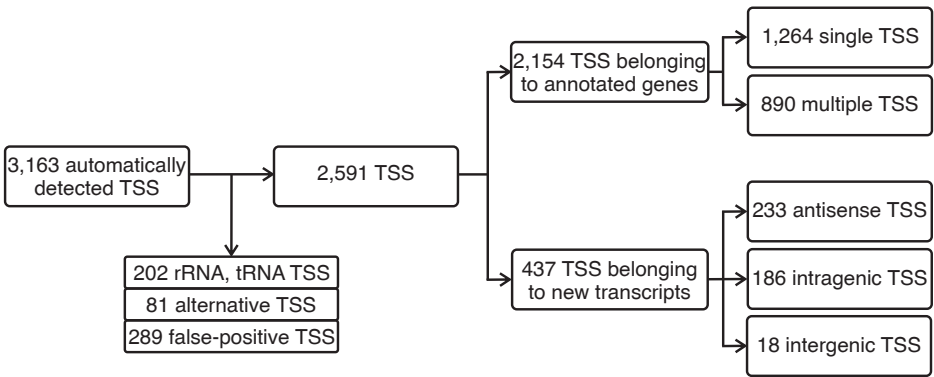

Figure 2 Classification of TSSs obtained with RNAseq. (a) Illustration of categories for TSS classification based on genomic context. The first TSS classification level is divided into two categories: TSSs that belong to annotated genes (gray shaded arrows) and TSSs that belong to new transcripts (black shaded arrows). TSSs belonging to annotated genes were classified into single TSSs or multiple TSSs. TSSs belonging to new transcripts were arranged into antisense, intragenic or intergenic TSSs. (b) Identification, filtering, and classification of TSSs. From the automatically detected TSSs those TSSs were removed that belong to rRNA or tRNA, false-positive, or alternative TSSs. 
genes $[8,37,38] . \sigma^{70}$ binds to specific promoter elements, called the -10 and a -35 region [8,39-42].

Thus, using the identified TSSs, it is possible to search for promoter motifs. For this search the web program Improbizer [43] was used to scan 60 bases upstream of each of the 2,591 identified TSSs. The conserved -10 motif "TAnnnT" was found in about $97 \%$ (2,522 in total) of the upstream sequences of the identified TSSs with a spacer (distance between -10 motif and TSS) of 3 11 nt (Figure 3, Additional file 1: Table S1). The motif as well as the distance of the motif to the TSS are similar to the published $\sigma^{\mathrm{A}}$ consensus promoter of C. glutamicum $[8,44]$.

Then, a -35 region was searched using the 2,522 upstream sequences with identified -10 motifs. As a further requirement for the identification of the -35 motif the position of the found motif was taken into account. Therefore, only a spacer length (distance between the -10 and -35 region) of $16-19$ nt was allowed [8]. Regarding these requirements 704 motifs could be determined showing a weakly conserved "ttgnca" motif (Figure 3, Additional file 1: Table S1).

This motif fits the previously published data for C. glutamicum $[8,44]$.

\section{Re-annotation of coding sequences}

The RNAseq data containing the TSSs turned out to be very useful for the correction of translational starts of coding sequence. Especially leaderless mRNAs were conducive for re-annotation. In total, the translational start codon positions of 205 genes were corrected. As a result of this re-annotation, 185 genes now encode leaderless mRNAs and the remaining 20 are genes containing $5^{\prime}$ UTRs (Additional file 1: Table S2). The TSSs of these leadered genes mapped within the particular gene but not on a start codon that is in-frame to the annotated stop codon, so that a leaderless mRNA for the appropriate gene could be excluded and a new, corrected translational start codon was searched downstream of the TSS. For this search, DNA sequences from the TSS position to the annotated stop codon of each of the 20 genes were scanned using the web program ORF finder [46] with ATG, GTG, CTG, and TTG as start codons and TGA, TAA, and TAG as stop codons. The identified and most meaningful gene starts for the 20 genes containing 5'-UTRs are listed in Additional file 1: Table S2.

For further RNAseq analyses, especially for the determination of 5'-UTRs, analysis of RBSs, identification of operons structures, and detection of novel transcripts, these re-annotated gene starts were used.

\section{Characteristics of 5'-UTRs and leaderless mRNAs}

The 2,147 TSS that were assigned to annotated, protein coding sequences were used for an analysis of the $5^{\prime}$ UTRs, the part of a transcript reaching from the TSS to the start codon. Quite surprisingly, 33\% (707 of 2,147) of the mRNAs in C. glutamicum were found to have no 5 '-UTR (5'-UTR length =0; Additional file 1: Table S3). These mRNAs were classified as leaderless in this study. This high fraction of leaderless mRNA do not show any significant preference to functional eggNOG [47] categories (data not shown). The analysis of initiator codons of such mRNAs in C. glutamicum revealed that all leaderless mRNAs have an AUG ( 79\%) or GUG ( 21\%) start codon.

Beside the many leaderless genes, there is a further accumulation of $5^{\prime}$-UTRs with lengths between 26 - $40 \mathrm{nt}$ (278 in total). These short leaders might only harbor a ribosome-binding site for translation.

Analysis of the distribution of the $5^{\prime}$-UTR lengths revealed that in general the number of $5^{\prime}$-UTRs within a certain bin decreased exponentially with increasing length (Figure 4). However, an interesting exception is represented by $805^{\prime}$-UTRs with a length of $1-10$ nt. These 5 '-UTRs do not provide enough sequence for a RBS and spacing to the initiation codon. Hence, the mechanism of translation initiation for such mRNAs and whether such transcripts result in functional proteins remains unclear.

A relatively high number of mRNAs contain $5^{\prime}$-UTRs longer than 100 nt (531 of 2,147 in total; Additional file 1: Table S3). It can be assumed that these long

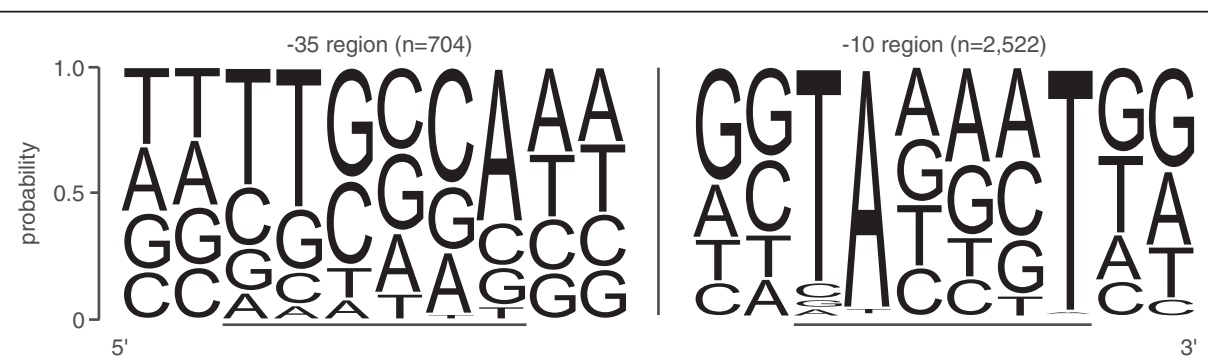

Figure 3 Distribution of nucleotides within the -10 and -35 regions of $C$. glutamicum $\sigma^{A}$ promoters. Relative occurrence of a nucleotide at a particular position is represented by the size of the nucleotide. The representation is based on 2,522 -10 and 704 -35 regions identified with Improbizer [43]. The core -10 and -35 regions are underlined. The sequence logo was created with Weblogo [45]. 


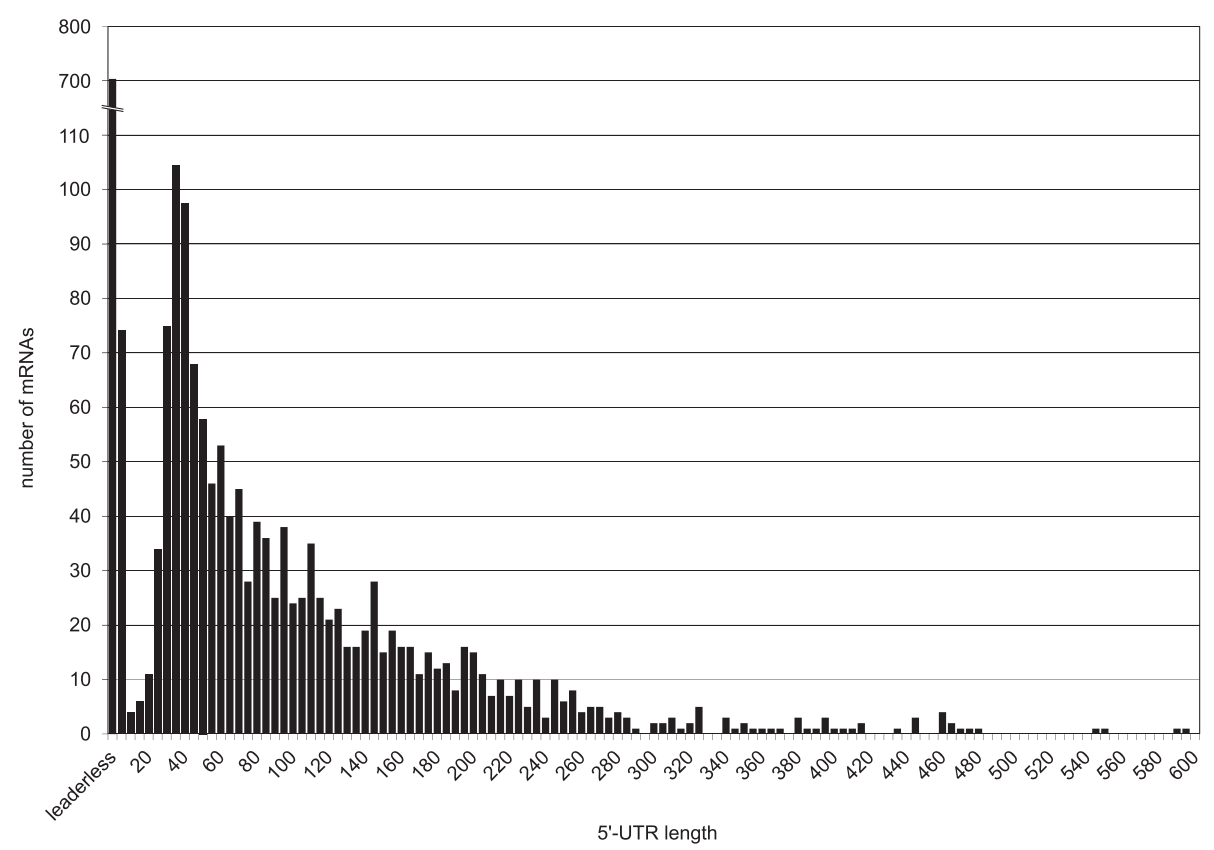

Figure 4 Distribution of 5'-UTR length of mRNAs belonging to annotated protein-coding genes in C. glutamicum. The distribution is based on 2,147 TSSs assigned to mRNAs. The bar labeled leaderless represents an UTR length of zero. The other bars represent UTR length in increments of five $(1-5,6-10,11-15$, etc.).

5 '-UTRs play a role in the translational regulation of their respective mRNAs through folding into secondary structures. Such cis-regulatory mechanisms of 5'-UTRs were previously described for many bacteria and can harbor sequences encoding leader peptides, riboswitches, RNA thermometers or binding sites for trans-encoded RNAs $[13,48,49]$.

To detect putative cis-regulatory $5^{\prime}$-UTR candidates, Rfam [50] database predicted regulatory regions within $5^{\prime}$-UTRs in C. glutamicum were compared with the RNAseq data obtained. Altogether, 16 regulatory regions were predicted for C. glutamicum and 13 of them matched the RNAseq data (Table 2). Supporting evidence for these riboswitches was provided by the annotation of the associated protein-coding regions. For example, the gene cg0083 encodes a predicted mononucleotide transporter and shows a putative flavin mononucleotide (FMN) riboswitch within the $5^{\prime}$-UTR for sensing flavin mono- and/or dinucleotides. Furthermore, the products of the genes $c g 1476$ (thiC), cg1655 (thiM), and cg2236 (thiE) are involved in thiamine metabolism and these genes contain putative thiamine pyrophosphate (TPP)-sensing riboswitches within their 5 '-UTRs. It is attractive to speculate that the two other genes with a predicted and validated TPP riboswitch, $\operatorname{cg} 0825$ encoding a putative short-chain oxidoreductase and cg1227, encoding part of an ABC transporter, are also involved in thiamine-dependent processes. Additionally, one RNA thermometer, the cspA mRNA
$5^{\prime}$-UTR, was observed within the 5'-UTR of $c g 0215$ that encodes a predicted cold-shock protein.

To identify possible secondary structures within the regulatory $5^{\prime}$-UTRs predicted by Rfam [50] and validated by RNAseq, the web server program RNAfold [51] was utilized. The sequences of the putative regulatory 5 '-UTRs including 15 bases of the coding region were used for the structure predictions. In all 13 cases, the 5 '-UTRs can fold into stable stem-loop structures, where the RBS is (partly) sequestered (Additional file 3: Figure S2).

It should also be mentioned here that small leader peptides within 5 '-UTRs of genes and operons involved in amino acid synthesis (cg3359/trpE, cg0303/leuA, cg1435/ilvB, and cg1129/aroF) were detected in C. glutamicum by RNAseq analysis [9]. These leader peptides are possibly involved in transcription attenuation and control the expression of the appropriated genes dependent on the amino acid level $[52,53]$.

\section{Analysis of ribosome binding sites}

Based on the 5'-UTRs of protein-coding genes, it is possible to analyze RBSs. Therefore, the frequency of purines ( $G$ and $A$ ) compared to that of pyrimidines ( $T$ and $C$ ) of each nucleotide within the $20 \mathrm{nt}$ upstream of the start codon was computed (Figure 5a) and showed an accumulation of purines (> 55\%) in the region between 6 to 18 bp upstream of the start codon with a peak around -11 relative to the first base of the start codon 
Table 2 Rfam predictions for regulatory regions in C. glutamicum compared to RNAseq data

\begin{tabular}{|c|c|c|c|c|c|c|c|c|c|}
\hline \multicolumn{5}{|l|}{ Rfam prediction ${ }^{a}$} & \multirow[b]{2}{*}{ Strand } & \multicolumn{4}{|c|}{ RNAseq identification $^{\text {b }}$} \\
\hline Name & ID & Start & End & Bit Score & & RNAseq & Start & End & Gene \\
\hline FMN riboswitch & RF00050 & 66,442 & 66,279 & 111.60 & - & observed & 66,438 & 66,198 & cg0083 \\
\hline cspA mRNA 5'-UTR & RF01766 & 186,399 & 186,766 & 60.19 & + & observed & 186,328 & 186,508 & $\operatorname{cg} 0215(\operatorname{csp} A)$ \\
\hline TPP riboswitch & RF00059 & 742,654 & 742,547 & 63.54 & - & observed & 742,651 & 742,490 & cg0825 \\
\hline ydaO-yuaA leader & RF00379 & 870,027 & 869,859 & 69.93 & - & observed & 870,047 & 869,853 & cg0936 (rpf1) \\
\hline TPP riboswitch & RF00059 & $1,127,774$ & $1,127,883$ & 51.03 & + & observed & $1,127,765$ & $1,127,874$ & cg1227 \\
\hline mini-ykkC RNA motif & RF01068 & $1,131,047$ & $1,131,094$ & 33.52 & + & not observed & - & - & - \\
\hline TPP riboswitch & RF00059 & $1,373,213$ & $1,373,103$ & 55.87 & - & observed & $1,373,210$ & $1,373,105$ & cg1476 (thic) \\
\hline SAM-IV riboswitch & RF00634 & $1,374,007$ & $1,374,123$ & 70.47 & + & observed & $1,374,005$ & $1,374,139$ & $\operatorname{cg} 1478$ \\
\hline TPP riboswitch & RF00059 & $1,544,490$ & $1,544,383$ & 52.11 & - & observed & $1,544,485$ & $1,544,390$ & cg1655 (thiM) \\
\hline yybP-ykoY leader & RF00080 & $1,550,030$ & $1,550,196$ & 43.71 & + & not observed & - & - & - \\
\hline yybP-ykoY leader & RF00080 & $2,043,157$ & $2,042,989$ & 49.13 & - & observed & $2,043,151$ & $2,042,955$ & cg2157 (terC) \\
\hline TPP riboswitch & RF00059 & $2,120,271$ & $2,120,383$ & 62.55 & + & observed & $2,120,271$ & $2,120,384$ & cg2236 (thiE) \\
\hline mraW RNA motif & RF01746 & $2,267,021$ & $2,266,916$ & 56.64 & - & observed & $2,266,932$ & $2,266,800$ & cg2377 (mraW) \\
\hline ydaO-yuaA leader & RF00379 & $2,292,467$ & $2,292,279$ & 59.37 & - & observed & $2,292,509$ & $2,292,267$ & cg2402 \\
\hline msiK RNA motif & RF01747 & $2,582,375$ & $2,582,317$ & 52.13 & - & observed & $2,582,404$ & $2,582,315$ & cg2708 (msik1) \\
\hline yybP-ykoY leader & RF00080 & $2,649,004$ & $2,648,890$ & 49.90 & - & not observed & - & - & - \\
\hline
\end{tabular}

${ }^{a}$ Name, ID, coordinates, and bit score of predicted regulatory 5'-UTRs for C. glutamicum were taken from the Rfam database [50].

${ }^{\mathrm{b}}$ Refers to detected TSSs and RNAseq ends relate to the last position of the $5^{\prime}-\mathrm{UTR}$. The secondary structures of the verified regulatory regions are shown in Additional file 3: Figure S2.

(Figure 5a) indicating a RBS within this region. A scan for a sequence motif within this region $(14-20$ bases upstream of the initiator codon; 928 sequences in total) using the web program Improbizer [43] confirmed this indication by detecting the conserved motif AGGag in about $92 \%$ of the 5 -UTR sequences (Figure 5c, Additional file 1: Table S4).

Next, the distance distribution between the RBS and the initiator codon was calculated by binning the length of the spacers. The distribution for all detected RBS revealed a spacing of $4-12 \mathrm{nt}$ as being the most common (>90\%), with $7.7 \pm 2.7 \mathrm{nt}$ as mean spacing (Figure $5 \mathrm{~b}$ ).

Here, we analyzed only ribosome sites that are located in the $5^{\prime}$-UTR of transcripts. RBSs within intercistronic regions were excluded from this analysis and will be addressed in more detail in future studies. However, the identified RBS motif AGGag can also be found within those intercistronic regions (data not shown).

\section{Identification of operon structures based on the whole transcriptome data set}

Due to the usage of the developed whole transcriptome protocol and the paired-end sequencing, as well as the primary 5 '-end data, we were able to identify operon structures in C. glutamicum and could assign genes to monocistronic transcripts, primary operons, and suboperons. Genes were assigned to a primary operon, if 15 or more combined read pairs connect neighboring genes. Thus, a primary operon builds a chain of co- transcribed genes. All remaining genes that could not be assigned to primary operons were categorizes as monocistronic transcripts. As RNAseq data from the whole transcriptome as well as data from the primary 5 '-ends were available and can be combined, in several cases we found that polycistronic operons have internal transcription starts and posterior genes might also form alternative sub-operons $[15,21]$ within the larger primary operon (Figure 6).

Altogether, 1,943 annotated genes could be assigned to 616 primary operons, including 565 sub-operon structures (Additional file 1: Table S5). Furthermore, this analysis showed that two-thirds of the estimated $\sim 3,000$ genes in C. glutamicum are transcribed as operons and one-third monocistronically (1,013 in total).

Additionally, the non-coding RNAs 6C RNA, and 4.5S RNA were identified as monocistronic transcripts, and the M1 RNA was assigned to a primary operon as it was found co-transcribed with annotated protein-coding genes. Furthermore, 8 tRNAs were also found to be co-transcribed with protein-coding genes (Additional file 1: Table S5). The remaining 87 genes that could not be allocated to primary operons or monocistronic transcripts had very low transcript levels (mean coverage below 1).

Generally, the quantity of operons decreases with increasing number of genes (Figure 7). Thus, only 9 primary operons contain 10 or more genes (Table 3), whereas 320 primary operons contain 2 genes. The largest primary operon identified here contains 16 genes 

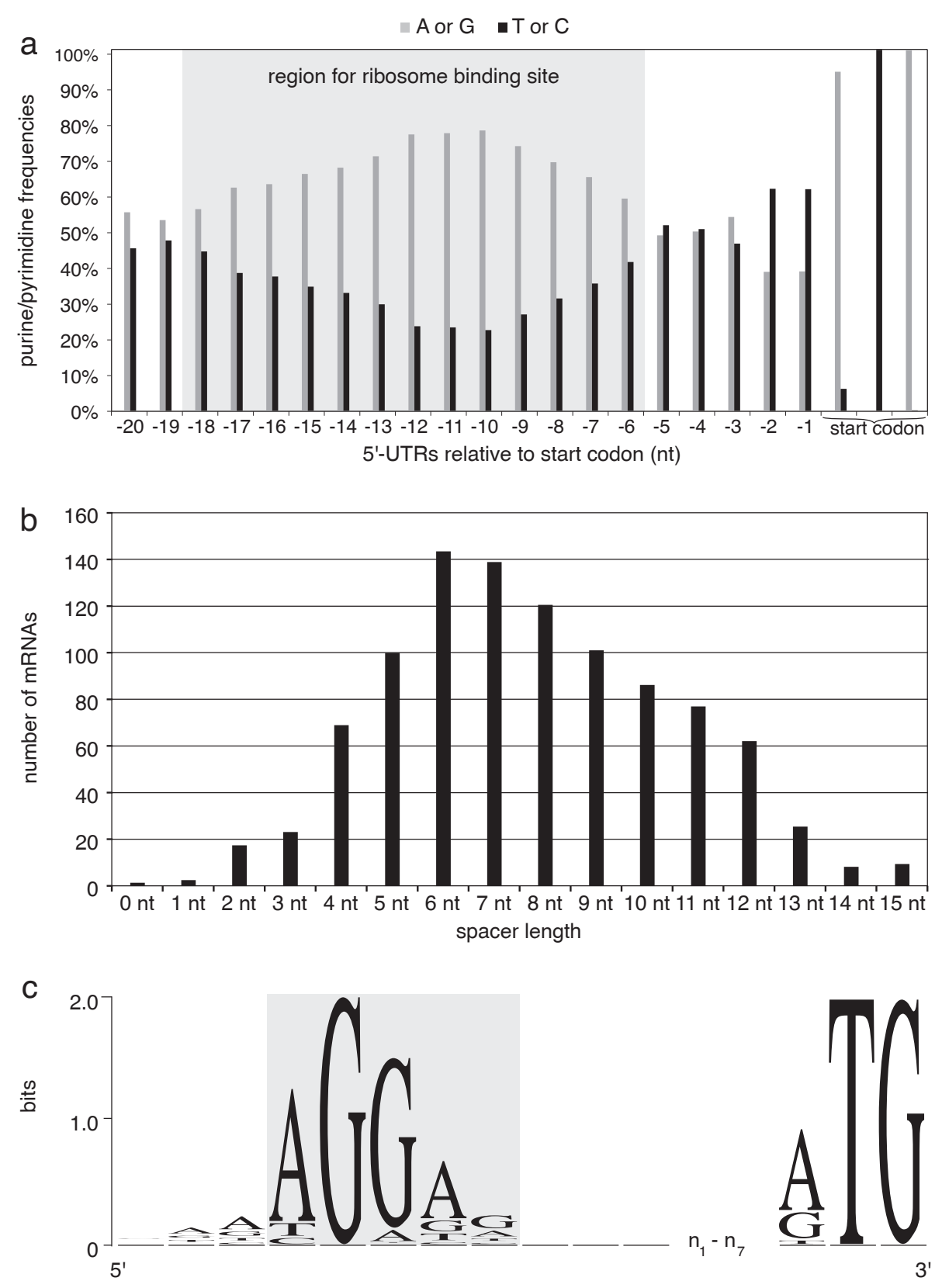

Figure 5 Analysis of ribosome binding sites in C. glutamicum. (a) Frequencies of purines (G or A) compared to frequencies of pyrimidines ( $T$ or $C$ ) within the first 20 bases (relative to the start codon) of 922 different $5^{\prime}$-UTRs. (b) Analysis of the spacing between the RBS and the start codon, based on all identified RBS motifs by Improbizer. (c) Information content within the identified RBS motif of C. glutamicum including three leading and lagging bases, a spacer of 1 - $7 \mathrm{nt}$, as well as the translational initiation codon. The $\mathrm{y}$-axis shows the information content (measured in bits). The analysis is based on 673 RBS motifs identified with Improbizer within a spacing of $7 \pm 3$ nt only. The sequence logo was created with Weblogo [45].

coding for proteins with diverse cellular functions (translation, ribosomal structure and biogenesis, transcription, replication, recombination and repair, carbohydrate transport and metabolism, and coenzyme transport and metabolism, according to eggNOG classification [47]). Upon manual inspection, the gene functions of this large transcription unit comprise riboflavin biosynthesis, protein modifying enzymes and the important $S$-adenosylmethionine (SAM) synthase, responsible for the synthesis of the main methyl group donor of the cell, SAM. It was apparent, that also the proteins of other cofactor biosyntheses are organized in long operons. This was the case for heme, cytochrome, folate and nicotinamide adenine dinucleotide (NAD). Other long operons contain functions in 


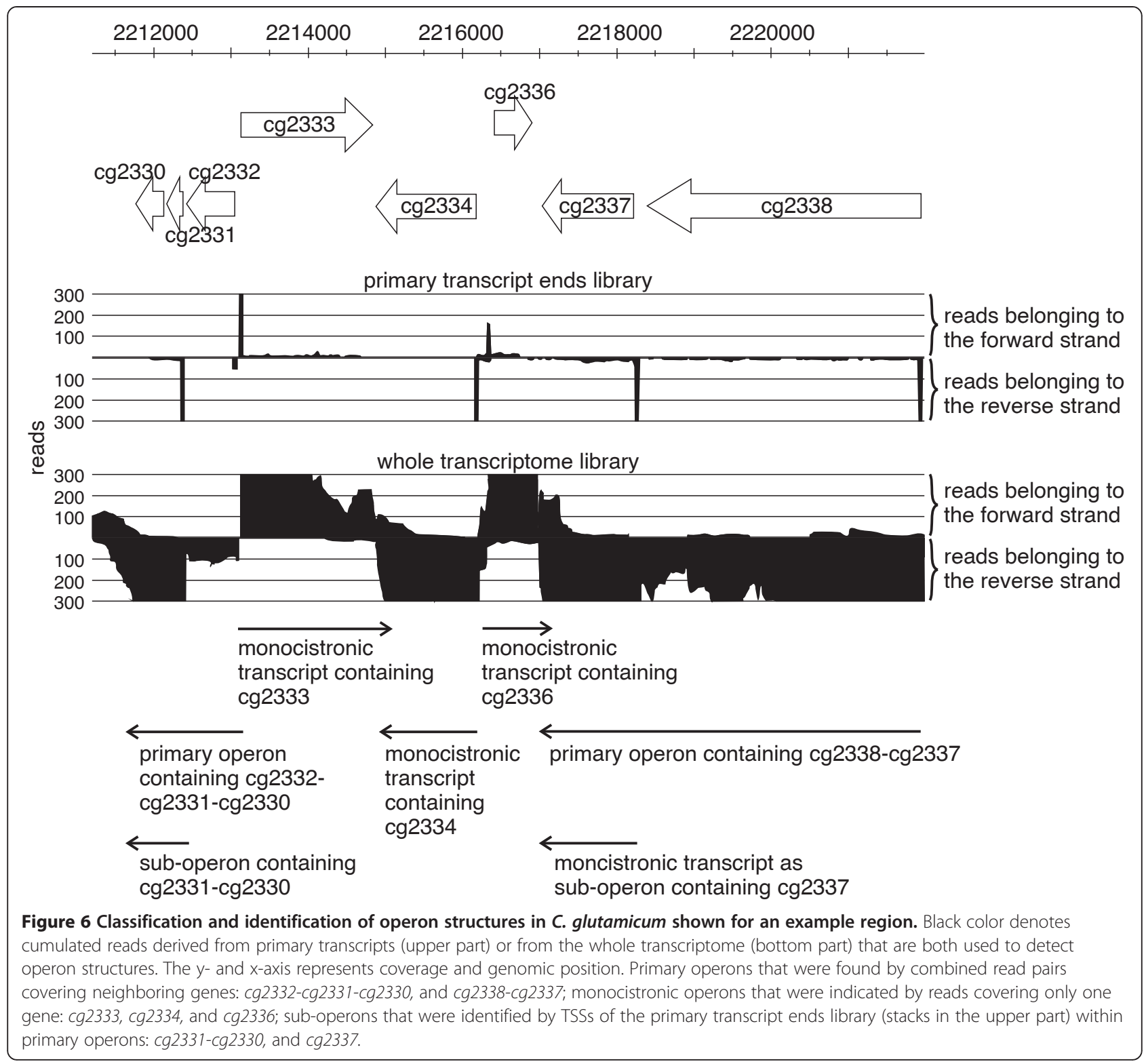

protein turnover (ribosomal proteins, chaperons, and the pupylation/proteasome machinery responsible for protein degradation).

\section{Description and classification of novel transcripts}

Besides the validation of already known genes, the obtained transcriptome data was mined to identify new transcripts that were so far unknown for C. glutamicum. For description of the start of a new transcript the primary $5^{\prime}$-end as well as the whole transcriptome data was used. For the determination of the 3 '-end of a new transcript the whole transcriptome data was applied. Due to the constraints used for the construction of the cDNA library, the data mostly contain transcripts that are larger than 200 nt. The identification and characterization of small RNAs in C. glutamicum using RNAseq was studied in detail separately [9].

Altogether, the newly found transcripts were classified into three categories: intergenic transcripts that are located between annotated genes, antisense transcripts that are located on the opposite strand of a transcripts and transcripts that start within a CDS.

In total, our data revealed 916 novel transcripts for C. glutamicum. Of these, 30 were identified as intergenic transcripts, 700 as antisense transcripts and 186 as intragenic transcripts (Additional file 1: Tables S6, S7 and S8).

For 18 of the 30 intergenic transcripts, a TSS was identified from the primary $5^{\prime}$-end data set. As intergenic transcripts might encode proteins, the sequences 


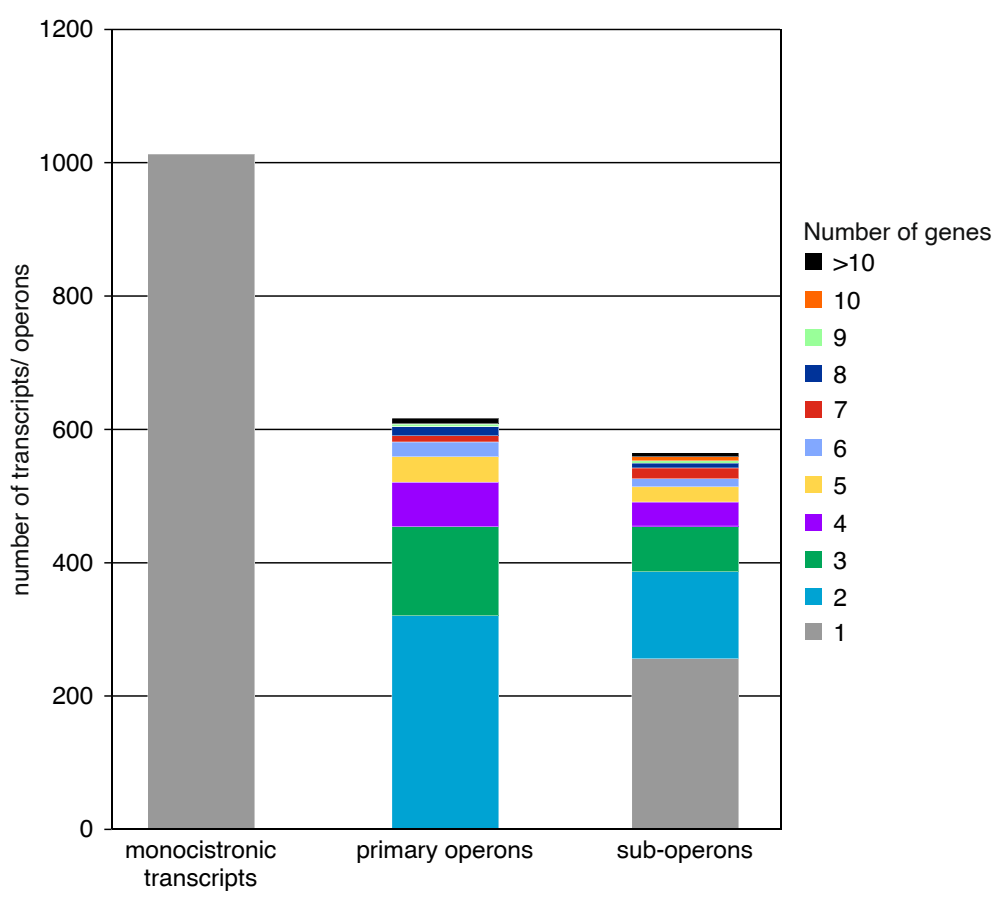

Figure 7 Analysis of the gene numbers in monocistronic transcripts, primary operons, and sub-operons in C. glutamicum. Operons differing in the number of genes are shown in different colors.

of these intergenic transcripts were scanned for open reading frames using the web program ORF finder [46], resulting in the identification of at least one open reading frame for 29 transcripts. In total, 20 of 29 identified open reading frames had at least one homologous protein in another species. Furthermore, to obtain additional evidence for encoded proteins within the new intergenic transcripts, a RBS was searched upstream of the longest predicted open reading frame. While one transcript was predicted to be leaderless, a potential RBS

Table 3 List of the largest identified primary operons ( $\geq 10$ genes)

\begin{tabular}{|c|c|c|c|c|}
\hline Genes & $\begin{array}{c}\text { Gene } \\
\text { number }\end{array}$ & Strand & TSS $^{\mathbf{a}}$ & eggNOG [47] classification of genes within primary operon ${ }^{b}$ \\
\hline $\begin{array}{l}\text { cg3011 to } \\
\text { cg3020 }\end{array}$ & 10 & - & detected & posttranslational modification, protein turnover, chaperones; unknown function \\
\hline $\begin{array}{l}\text { cg0593 to } \\
\text { cg0604 }\end{array}$ & 11 & + & detected & translation, ribosomal structure and biogenesis (ribosomal proteins) \\
\hline $\begin{array}{l}\operatorname{cg} 1683 \text { to } \\
\operatorname{cg} 1693\end{array}$ & 11 & - & detected & 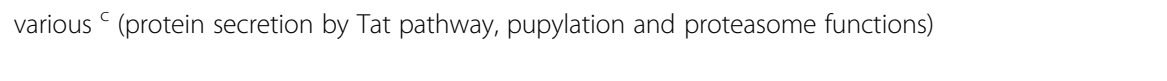 \\
\hline $\begin{array}{l}\operatorname{cg} 2578 \text { to } \\
\operatorname{cg} 2589\end{array}$ & 11 & - & detected & various (proline and NAD biosynthesis) \\
\hline $\begin{array}{l}\text { cg0414 to } \\
\text { cg0424 }\end{array}$ & 11 & + & detected & $\begin{array}{l}\text { signal transduction mechanisms; cell wall/membrane/envelope biogenesis; amino acid transport and } \\
\text { metabolism; unknown function (cell envelope, glycan structures) }\end{array}$ \\
\hline $\begin{array}{l}\text { cg2974 to } \\
\text { cg2987 }\end{array}$ & 13 & - & detected & various (lysyl-tRNA synthase, folate biosynthesis, nucleotide salvage) \\
\hline $\begin{array}{l}\text { cg0510 to } \\
\text { cg0524 }\end{array}$ & 14 & + & detected & $\begin{array}{l}\text { carbohydrate transport and metabolism; coenzyme transport and metabolism; posttranslational } \\
\text { modification, protein turnover, chaperones; inorganic ion transport and metabolism; unknown function } \\
\text { (heme and cytochrome c biosynthesis) }\end{array}$ \\
\hline $\begin{array}{l}\operatorname{cg} 2363 \text { to } \\
\operatorname{cg} 2377\end{array}$ & 15 & - & detected & $\begin{array}{l}\text { cell cycle control, cell division; cell wall/membrane/envelope biogenesis; unknown function } \\
\text { (peptidoglycan biosynthesis, cell division) }\end{array}$ \\
\hline $\begin{array}{l}\text { cg1792 to } \\
\text { cg1807 }\end{array}$ & 16 & - & detected & various (riboflavin biosynthesis, protein modification, S-adenosylmethionine synthesis) \\
\hline
\end{tabular}

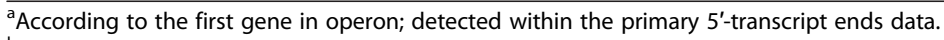

${ }^{b}$ Functional classes based on manual inspection are included in brackets.

${ }^{\mathrm{c}} \mathrm{Genes}$ within one primary operon are classified to more than 5 different eggNOG [47] functions. 
was identified for 13 of the 28 remaining transcripts (Additional file 1: Table S6).

In total, 700 new transcripts were classified as antisense transcripts. Of these, 696 are located in antisense orientation to one or more annotated protein-coding sequences. The remaining four antisense transcripts are located on the opposite strand of other transcripts (Additional file 1: Table S7). Yet, only for 233 of those antisense transcripts a clear TSS could be identified. The remaining transcripts probably arise from $\sigma^{\mathrm{A}}$-independent transcription events occurring only under stress conditions since they were found in the total transcriptome library but not in the $5^{\prime}$-enriched data set.
Transcript ends and predicted rho-independent terminators

In addition to the analysis of transcription start sites and operon structures, we also examined the 3 '-transcript ends and compared those to rho-independent terminators predicted by TransTermHP [54]. Therefore, we extracted the TransTermHP predictions for all monocistronic transcripts and the last gene within the defined primary operons. Overall, we obtained 1,383 predicted intrinsic terminators and for only 320 (23.1\%) of these a clear transcript end could be determined, based on the following criteria: the mean coverage of the last 5 bases before the first terminator stem must be at least 11 and the ratio of this mean coverage and

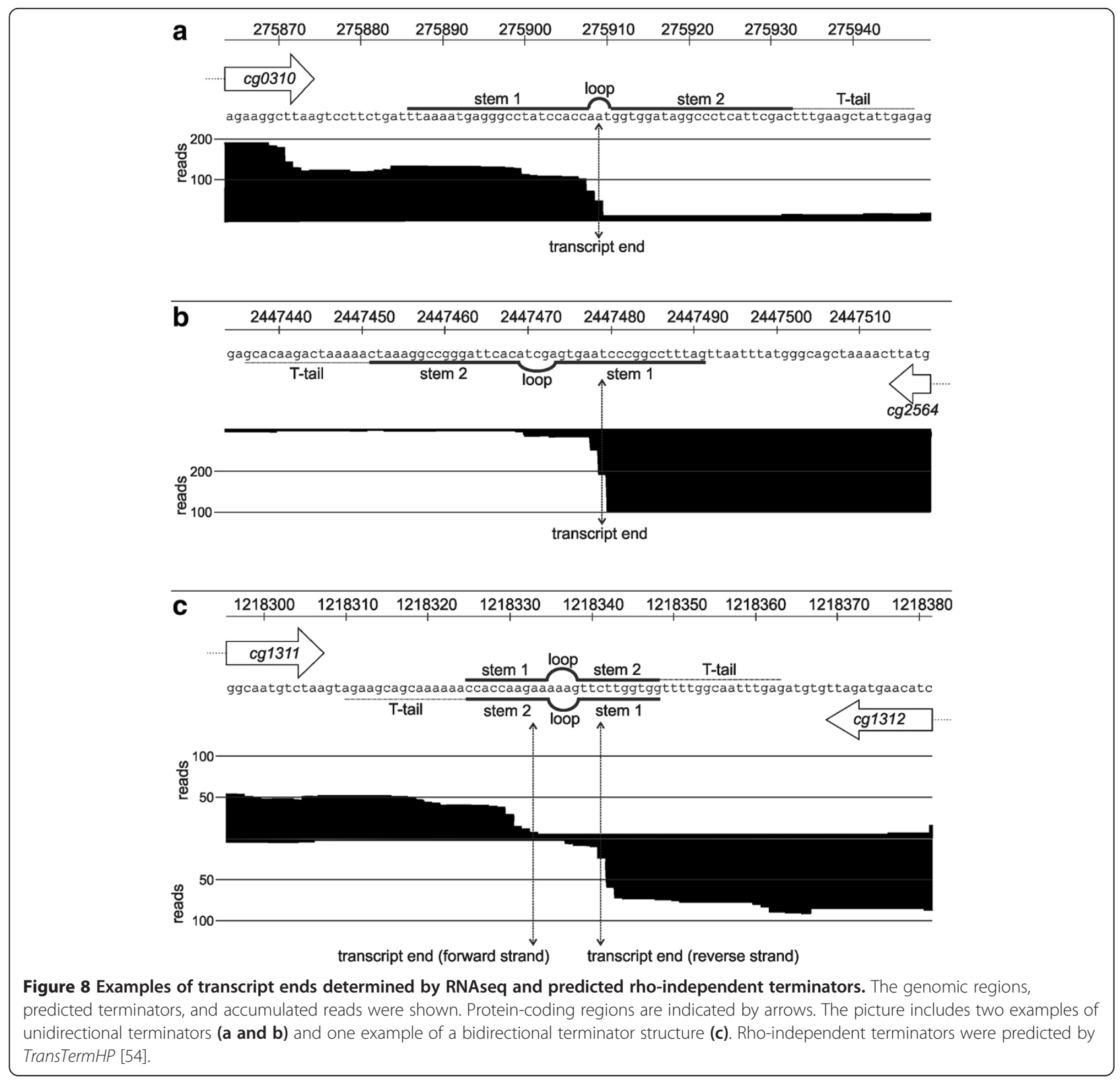


the coverage of a given base within the terminator must be $\geq 5$. Of the remaining 1,063 predicted terminators, 290 (21\%) transcript ends were insufficiently covered and 773 (55.9\%) displayed only a gradual decrease in coverage. For the 320 predicted rho-independent terminators we found that the majority of transcript ends were located within stem 1 of the predicted rho-independent terminator and the minority within the loop, stem 2, or T-tail (72.2\% within stem $1,18.4 \%$ within the loop, $7.5 \%$ within stem 2 , and $1.9 \%$ within the T-tail; Figure 8, Additional file 1: Table S9). It was surprising that only a low number of 3 '-ends were located in the T-tail.

\section{Discussion}

This study presents the first comprehensive transcriptome analysis of Corynebacterium glutamicum. The complete genome sequence of C. glutamicum ATCC 13032 and its annotation were published in 2003 [4,5], but knowledge on transcriptional organization, promoter elements, and non-coding transcripts was known only for a few selected examples.

The use of RNAseq to analyze and characterize the transcriptomes in prokaryotic as well as in eukaryotic cells has been exploited in recent times [15,17,26,55-58]. While commercial RNAseq kits were used in most of the above-mentioned studies, here, we introduce two improved RNAseq library preparation protocols which provide to the possibility to customize each step as required. The described approach can easily be adapted for other species.

For the description of the transcriptome profile of C. glutamicum, we combined the results of two RNAseq protocols, one that addresses the primary and the other the whole transcriptome. Combining both data sets, we were able to report defined 5 ' - and 3 '-ends of annotated transcripts, novel transcripts, and operon structures. The sequencing of a library that contained enriched primary transcript ends enabled a mapping of 2,591 TSSs that represented the basis for the analysis of promoter motifs, $5^{\prime}$-UTRs, RBSs and operon structures.

\section{$\sigma^{A}$-dependent promoters in C. glutamicum}

The search for recurring motifs at the 2,591 TSSs revealed a -10 region (TAnnnT) and a less conserved -35 region (ttgnca) of the sigma factor $\sigma^{\mathrm{A}}$-dependent promoter in C. glutamicum. This primary sigma factor is present in all known eubacteria $[8,37,38]$ and in C. glutamicum its reported recognition sequence consists of two hexamers, TTGNCA and TANANT, located around the positions -35 and -10 relative to the TSS [8] both of which closely match our results. It was previously observed that C. glutamicum promoters do not have well conserved -35 regions $[8,44]$. The weak conservation of the -35 motif in C. glutamicum can be explained by the fact that there is a high number of promoters with an extended -10 promoter element, TGnTATAAT $[41,59,60]$, able to specify the full functionality for sigma factor recognition. In addition to the house-keeping $\sigma^{\mathrm{A}}$ and the primary-like $\sigma^{\mathrm{B}}$, C. glutamicum possesses five additional sigma factors of the ECF family $\left(\sigma^{\mathrm{C}}, \sigma^{\mathrm{D}}, \sigma^{\mathrm{E}}, \sigma^{\mathrm{H}}\right.$, and $\left.\sigma^{\mathrm{M}}\right)$, each responsible for recognizing promoters of genes involved in specific functions and stress responses [8]. These sigma factors might be responsible for recognition of promoters under stress conditions that were analyzed only in the whole transcriptome sample. Since the RNA sample for 5 '-enriched library was generated from unstressed conditions, promoter analyses were only performed for putative housekeeping promoters. Additional analyses of these promoters and their motifs will be performed in future studies (Albersmeier et al., in preparation).

The identification of TSSs also revealed that 365 genes contain multiple TSSs (up to six) that result in different lengths of the $5^{\prime}$-UTR. The occurrence of multiple TSSs can be explained by the presence of multiple promoters. The use of multiple promoters might be helpful for adaption to nutritional signals, with one promoter ensuring a constitutive gene expression and the others increased transcription in presence of specific stimuli [61].

\section{Characterization of 5'-UTRs indicate novel regulatory elements}

Our analysis confirmed the existence of $135^{\prime}$-UTRs that have been predicted by the Rfam database [50], among them several riboswitches (e.g. 5 thiamin pyrophosphatesensing riboswitches as 5'-UTRs of cg0825, cg1227, cg1476, cg1655, and cg2236, one S-adenosylmethionine-sensing riboswitch as $5^{\prime}$-UTR of $c g 1478$, and one flavin mononucleotide-sensing riboswitch as $5^{\prime}$-UTR of $c g 0083)$. Riboswitches control gene expression at the mRNA level by undergoing conformational changes. A change is usually induced upon binding of small molecules that provide chemical moieties to interact with nucleic acids, often products of metabolic pathways they thereby regulate $[48,52,53]$. The occurrence of riboswitches in the $5^{\prime}$-UTR region of metabolic genes that directly bind diverse metabolites and influence both at transcriptional and translational level have been described for Gram-positive and Gram-negative bacteria $[52,53,62,63]$. For C. glutamicum, the existence and function of these riboswitches is indeed likely as some genes containing putative riboswitches within their $5^{\prime}$-UTR are annotated as enzymes with a clearly associated metabolic function. In other cases, the existence of a riboswitch of a certain class will be helpful in identification of the functional context of this gene. Beside the metabolite-sensing riboswitches, one RNA thermometer (within the $5^{\prime}$-UTR of $c g 0215$ ) could also be validated by the annotation of its gene as putative 
cold-shock protein $(\operatorname{csp} A)$. In $E$. coli the $\operatorname{csp} A$ thermosensor is described to modulate the translation of the $\operatorname{csp} A$ mRNA (encoding a cold-shock protein) more efficiently at low temperatures than at higher temperatures, resulting in the adaption of cellular mechanisms at different temperatures as a cold-shock response [64]. The structural prediction of the RNA thermometer within the $5^{\prime}$-UTR of $\operatorname{cg} 0215$ and the cold-shock protein encoded by cg0215 mRNA indicate a similar function of the putative RNA thermometer in C. glutamicum, although this remains to be proven experimentally. Moreover, a wealth of candidates that might harbor novel cis-regulatory RNA structures was also identified. For example, genes involved in the metabolism of amino acids (e.g. arginine, cysteine, histidine, and methionine) contain $5^{\prime}$-UTRs longer than $60 \mathrm{nt}$ that might contain riboswitch-like structures (e.g. cg2305/hisD [65]). Previously, several RNA elements have been described to regulate amino acid metabolism genes in bacteria $[52,53,66]$. Such cis-regulatory elements allow a fine-tuning of gene expression [67], and this might be advantageous for fast-growing, metabolically versatile bacteria like C. glutamicum. Beside the occurrence of riboswitches and RNA thermometers within the 5'-UTRs of mRNAs, stem-loop structures of $5^{\prime}$-UTR which have a protective effect against mRNA degradation play a role in mRNA decay $[68,69]$. This might also be an explanation for the relatively high number of $5^{\prime}$-UTRs longer than $60 \mathrm{nt}$ in C. glutamicum.

\section{Abundance of leaderless mRNAs in C. glutamicum}

Approximately $33 \%$ of mRNAs were identified to be leaderless in C. glutamicum. Previous transcriptional studies have revealed $\sim 1.5 \%$ (26 of 1,907) leaderless mRNAs in Helicobacter pylori [15], 28\% (53 of 192 with a 5'-UTR < $11 \mathrm{nt}$ ) in Streptomyces coelicolor [32], and $\sim 4.7 \%$ (8 of 170 with a $5^{\prime}$-UTR $<11$ nt) in Pseudomonas putida KT2440 [26], so that the fraction of leaderless transcripts (707 of 2,147 mRNAs) in C. glutamicum is the highest described in any bacterium. It is already known that leaderless transcripts, although occurring in many taxonomic branches of bacteria, are especially abundant in Gram-positives [70]. Interestingly, it was previously found that archaeal genes are commonly expressed as leaderless transcripts [71,72], although there are also archaea which carry mainly long $5^{\prime}$-UTRs [73]. However, it is obscure whether there is a similarity between archaeal transcription and the transcriptional machinery of C. glutamicum since the transcription in archaea resembles more closely that in eukaryotes [74].

The translational mechanism of leaderless transcripts and the recognition of such mRNAs without a $5^{\prime}$-UTR and a canonical RBS by the ribosomes were unknown for a long time. However, in E. coli it was shown that the AUG start codon and the initiator tRNA can promote stable binding between the mRNA and the ribosome $[70,75]$, and that leaderless transcripts have a preference to $70 \mathrm{~S}$ ribosomal monomers over $30 \mathrm{~S}$ subunits, proposing an alternative translation initiation pathway for such mRNAs [76]. The work presented here revealed AUG ( $79 \%)$ and GUG ( 21\%) as initiation codon for leaderless transcripts in C. glutamicum. Although the mechanism, by which GUG can replace AUG in leaderless translation is not clear, it is described that GUG might function as start codon in leaderless transcripts also in other organisms, potentially providing a lower translational level $[32,70]$. It can be assumed that leaderless transcripts might be remnants of ancestral mRNAs, because heterologous leaderless mRNAs derived from bacteria can be reliably translated in archaea and eukaryotes [70]. The commonality and simplicity in the translation initiation on leaderless mRNAs in archaea, bacteria, and eukaryotes is also indicative for an early evolutionary origin of such a translation mechanism [77].

\section{RNAseq data reveal AGGag as ribosome binding site for C. glutamicum}

Based on our data, it was possible to obtain the most accurate and comprehensive analysis of the RBS in C. glutamicum so far. Our data show an increase in the fraction of purines $(>55 \%)$ in the region between the 6th and 18th nt upstream of the initiation codon, indicating a recognition region for the ribosome [28]. The search for a motif within this region identified the conserved motif AGGag in about $89 \%$ of 5 '-UTR sequences in C. glutamicum that represents the reverse complement of the 3'-terminus of the 16S rRNA. These discoveries fit very well to previously published data for $E$. coli [28,78-80]. Beside the initiation codon, the translation efficiency is also dependent on the hybridization of the RBS of the mRNA to the 3 '-end of the 16S rRNA, and thus, on its sequence conservation $[81,82]$. It was shown that translation can be increased using optimal RBS that are perfectly complementary to the 3 -terminus of the $16 \mathrm{~S}$ rRNA $[81,82]$. Therefore, it is likely that the most conserved bases from the 1st to the 3rd position (AGG) within the RBS motif AGGag in C. glutamicum are essential for the translation initiation mechanism, whereas the remaining bases modulate translation efficiency. The spacing between RBS and start codon was also shown to have a strong effect on translation, suggesting that the physical distance between the 3 '-terminus of the $16 \mathrm{~S}$ rRNA and the anti-codon of the initiator fMet-tRNA $[79,83]$ is of importance. In C. glutamicum, a spacing between $4-12$ nt was found to be the most common (> 90\%). The calculated mean spacing of $7.7 \pm 2.7 \mathrm{nt}$ fits 
well to the number derived for E. coli (mean spacing of $6.9 \pm 2 \mathrm{nt}$ ) [84]. Therefore, approx. 5 - $10 \mathrm{nt}$ is likely to be the "optimal" spacing.

\section{Revealing operon structures for C. glutamicum}

In this RNAseq approach, about $97 \%$ of the annotated genes were found to be transcribed under at least one of the nine different growth conditions used. Besides validating the annotated coding sequence, the genes could furthermore be assigned to 616 primary operons or to 1,013 monocistronic transcripts. Genes that are located next to each other and are transcribed coordinately from a single promoter form a genetic unit, an operon $[27,85]$. Due to the resolving power of RNAseq, executed on $5^{\prime}$-ends of native transcripts, it was furthermore found that 565 sub-operons are present. The existence of additional sub-operons was previously described for different bacteria: for example, the mapping of TSSs in Helicobacter pylori identified 337 primary operons, 126 sub-operons, and 66 monocistrons within the primary operons [15], whereas in Mycoplasma pneumoniae 341 identified operons could be divided into 447 smaller alternative transcription units by analyzing transcription at 173 different conditions [21]. The use of internal TSSs greatly increases the transcriptomic complexity and regulatory capacity $[22,86]$. Furthermore, other transcriptomic analyses revealed a condition-dependent modulation of operon structures. In such cases, a gene assigned to a polycistron in one condition can be transcribed as a monocistron in another condition [21,87] suggesting switchable operon structures [22].

In addition to the primary operons, we found that a third of the protein-coding regions in C. glutamicum are located on transcripts that are transcribed monocistronic. This large number indicates that in general, the advantage to regulate each gene individually, e.g. by transcription factors, outweighs the advantage of coordinated expression as part of a polycistron as well as the drawback of an increasing "regulatory burden" associated with an increasing number of monocistrons [67].

On the other hand, we found that genes involved in the synthesis of enzyme cofactors or in protein metabolism (from translation to degradation in the proteasome machinery) are organized in long operons. This finding might be interpreted that especially in these cases coordinated expression is the major organizational force.

\section{Detection of novel transcripts unveil a huge amount of antisense transcription}

Our data revealed 916 transcriptionally active, but yet unknown regions for C. glutamicum. Out of them, 30 were identified as intergenic, 186 as intragenic, and 700 as antisense transcripts (Additional file 1: Tables S6, S7 and S8). The intergenic transcripts most probably represent genes by themselves, either encoding small proteins or being a non-coding RNA. Small, noncoding RNAs are widely distributed in bacteria and also present in high numbers in C. glutamicum. Since the small RNA fraction of the bacterial transcriptome requires special preparation protocols for analysis by RNAseq, it is not properly represented in the data sets obtained here and subject of another study that addresses the small RNA fraction of the C. glutamicum transcriptome [9].

Quite a high number of intragenic TSSs (186 in total) that mapped within an annotated gene were determined for C. glutamicum. Yet, it is not clear whether this TSSs result in alternative, shorter proteins, novel proteincoding or non-coding genes. Such internal TSSs were also described for H. pylori [15]. Furthermore, such internal TSSs were also found in viruses and in human, producing a second, shorter gene product [88-90]. It is speculated that such internal transcripts that might harbor multi-functional, possibly regulatory regions increase the genomic information content [90].

Furthermore, 700 new antisense transcripts were identified for C. glutamicum. Yet, only for 233 of those a TSS was mapped. It is likely that the remaining transcripts arise from sigma factor $\sigma^{\mathrm{A}}$-independent transcription present only under stress conditions since they appeared in the library that included RNA from stress conditions but not in the other that was built from normal growth conditions. These antisense transcripts are expected to be non-coding RNAs and may have regulatory roles in gene expression. Strand-specific transcriptome sequencing has led to the description of massive amounts of antisense RNAs in various bacteria, eukaryotes, and archaea, and might represent a common form of regulation within all domains of life [22,91].

Antisense RNAs act on gene expression by a variety of different mechanisms. On one hand, they can hybridize to a part or the whole sense transcript, causing structural changes in the target affecting transcription termination (attenuation), introduce or block cleavage sites for ribonucleases (RNA cleavage), or have an effect on translation of the target gene by blocking or releasing the RBS (translation block) [91]. On the other hand, their transcription can alter sense transcription by interference, suppressing the production of the transcript due to polymerase collisions (transcription interference) [91]. The use of antisense RNAs for controlling gene expression allows an additional and tight regulation of target genes [91], and permits adaptation of gene expression to more different conditions. For C. glutamicum, the function and the regulation of expression of these antisense RNAs is hitherto unknown, but represent an interesting research target for future studies. 


\section{Transcript ends and predicted rho-independent terminators}

Rho-dependent and -independent termination are principal mechanisms by which bacterial transcription units are defined [92]. Intrinsic, rho-independent terminators are composed of a hairpin structure with $\mathrm{G}+\mathrm{C}$-rich stem followed by a T-stretch [93]. It is proposed that the RNA polymerase complex is destabilized after transcription and formation of the hairpin structure and that the transcribed RNA molecule is released within the T-stretch region [92,94].

The RNAseq data obtained here shows that transcription termination in C. glutamicum seems to take place within the T-tail region in only a minor number of transcripts. The most 3 '-ends of transcripts were found in the first $\mathrm{G}+\mathrm{C}$-rich region of the intrinsic terminator. This observation is contradictory to the literature $[92,94]$. A possible explanation might be an RNA processing within the stem structure of the terminator by RNases that cleave double-stranded RNA molecules. For $E$. coli it was shown that a read-through of the int gene (encodes a protein involved in site-specific recombination) leads to a formation of an RNase III cleavage site within the terminator to regulate the expression of this gene [95]. Since the chosen criteria were a sharp dropoff in the number of reads within the terminator, we might have selected for transcripts in which processing of double stranded RNA occurred. Another explanation for the transcript ends within the terminator stem might be that a cruciform structure formed on the DNA within the terminator region displaces the RNA polymerase thereby terminating transcription in the region of the terminator stem. Although it was shown in vitro that $A+T$-rich sequences can more easily form cruciform structures than $\mathrm{G}+\mathrm{C}$-rich sequences, it was never shown in vivo [96].

However, we cannot exclude that the use of the stemloop DNA adapter with seven wobble bases did not hybridize well in such stem-loop regions and therefore did not reach a perfect resolution of 3 '-transcript ends. To prove or disprove this and to get a better resolution of the 3'-transcript ends, other library preparation methods might be used. Suitable methods are already described for eukaryotic polyA-RNA: RNA-PET (RNApaired end tagging). In RNA-PET the $5^{\prime}$ - and $3^{\prime}$-end tags of full-length cDNA fragments are fused and subsequently sequenced [97].

It should be mentioned that for about $80 \%$ of the predicted rho-independent terminators for C. glutamicum no clear transcript end could be determined, either because of insufficient sequence coverage or because of a gradual and slow transcription drop-off. This indicates that the connection between RNAseq analysis and 3 '-ends or the transcripts or the processes involved in rho-independent termination in C. glutamicum or more general in actinobacteria are not yet fully understood.

\section{Conclusions}

In this study we have created the C. glutamicum transcriptome profiles using an improved RNAseq method. The generated transcriptome data reported in this manuscript is strand-specific and allows novel insights into the transcriptomic organization and a comprehensive discovery of novel transcripts from intergenic and antisense regions throughout the genome. We were able to map and describe operon structures for this bacterium and classified more than $70 \%$ of the new transcripts as antisense transcripts. We identified more than 2,000 TSSs supported by promoter search of the housekeeping sigma factor $\sigma^{\mathrm{A}}$ and re-annotated more than 200 gene starts. Surprisingly, we observed that about 33\% of the mRNAs in C. glutamicum are leaderless transcripts without any preference for functional categories of the proteins encoded by these transcripts. In this sense, it appears to be the ultimate tool for an information-rich genome annotation. The transcriptomic data established in this study deliver an enormous amount of information on various subjects and opens many new fields to be further investigated. Among these are hitherto unknown transcriptional mechanisms in C. glutamicum and transcription-based regulation on the transcriptional as well as on the translational level.

\section{Methods}

\section{Bacterial strains, oligonucleotide and culture media}

The bacterial strain used in this study is Corynebacterium glutamicum ATCC 13032. The oligonucleotides used are listed in Table 4. For shaking flask cultivation, C. glutamicum was grown in the complex medium LB (lysogeny broth) or in the chemically defined medium CGXII [98], but containing only $2 \%$ glucose (instead of $4 \%$ ) and $30 \mathrm{mg} \mathrm{l}^{-1}$ instead of $0.03 \mathrm{mg} \mathrm{l}^{-1}$ protocatechuic acid, in $250 \mathrm{ml}$ shaking flasks at $30^{\circ} \mathrm{C}$ and $300 \mathrm{rpm}$. The fermenter minimal medium was a derivate of CGXII medium containing $25 \mathrm{~g} \mathrm{l}^{-1} \mathrm{NH}_{4}\left(\mathrm{SO}_{4}\right)_{2}, 1 \mathrm{~g} \mathrm{l}^{-1} \mathrm{KH}_{2} \mathrm{PO}_{4}, 0.25 \mathrm{~g} \mathrm{l}^{-1}$ $\mathrm{MgSO}_{4}, 10 \mathrm{mg} \mathrm{l}^{-1} \mathrm{FeSO}_{4} \cdot 7 \mathrm{H}_{2} \mathrm{O}, 10 \mathrm{mg} \mathrm{l}^{-1} \mathrm{MnSO}_{4}$. $\mathrm{H}_{2} \mathrm{O}, 1 \mathrm{mg} \mathrm{l}^{-1} \mathrm{ZnSO}_{4} \cdot 7 \mathrm{H}_{2} \mathrm{O}, 0.2 \mathrm{mg} \mathrm{l}^{-1} \mathrm{CuSO}_{4} \cdot 5 \mathrm{H}_{2} \mathrm{O}$, $0.02 \mathrm{mg} \mathrm{l}^{-1} \mathrm{NiCl}_{2} \cdot 6 \mathrm{H}_{2} \mathrm{O}, 10 \mathrm{mg} \mathrm{l}^{-1} \mathrm{CaCl}_{2} \cdot 2 \mathrm{H}_{2} \mathrm{O}$, $2 \mathrm{mg} \mathrm{l}^{-1}$ biotin, $30 \mathrm{mg} \mathrm{l}^{-1}$ protocatechuic acid, and $4 \%$ glucose. For cultivation of C. glutamicum in a fermenter system, pre-cultures were grown in CGXII minimal medium in shaking flasks. The fermenter was then inoculated with pre-culture corresponding to a start $\mathrm{OD}_{600} \mathrm{~nm}$ (optical density) of 0.2. The fermentations were performed in 11 Biostat Q bioreactors (Sartorius, Göttingen, Germany) at $\mathrm{pH} 7,30^{\circ} \mathrm{C}$ and $30 \%$ dissolved oxygen. 


\begin{tabular}{|c|c|}
\hline Name & Sequence $\left(5^{\prime}-3^{\prime}\right)$ \\
\hline RNA adapter & $\begin{array}{l}\text { CCCUACACGACGCU } \\
\text { CUUCCGAUCGAG }\end{array}$ \\
\hline stem-loop DNA adapter & $\begin{array}{l}\text { AGATCGGAAGAGAG } \\
\text { ACGTGTGCTCTTCCGA } \\
\text { TCTNNNNNNN }\end{array}$ \\
\hline amplification primer 1 & $\begin{array}{l}\text { AATGATACGGCGACC } \\
\text { ACCGAGATCTACACT } \\
\text { CTITCCCTACACGAC } \\
\text { GCTCTTCCGATCGAG }\end{array}$ \\
\hline amplification primer 2 & $\begin{array}{l}\text { CAAGCAGAAGACGG } \\
\text { CATACGAGATCGTGA } \\
\text { TGTGACTGGAGTTCA } \\
\text { GACGTGTGCTCTTCCGATCT }\end{array}$ \\
\hline
\end{tabular}

All oligonucleotides used in this work were synthesized at Metabion International AG (Martinsried, Germany).

\section{Growth conditions for cell sampling}

To obtain the RNA for enrichment of primary transcripts as well as for the whole transcriptome, C. glutamicum cells were grown in shaking flasks in complex LB medium and defined CGXII medium as described above and harvested in the exponential growth phase.

For the whole transcriptome sample, C. glutamicum cells were additionally grown in shaking flasks in defined CGXII medium and subjected to different stresses at an $\mathrm{OD}_{600 \mathrm{~nm}}$ of 10. High salt stress: addition of sodium chloride to a final concentration of $10 \%$. Heat shock stress: shift of the temperature from $30^{\circ} \mathrm{C}$ to $50^{\circ} \mathrm{C}$. Cold shock stress: temperature shift from $30^{\circ} \mathrm{C}$ to $4^{\circ} \mathrm{C}$. Alcohol stress: addition of ethanol to a final concentration of $10 \%$. Oxidative stress: addition of diamide to a final concentration of $2 \mathrm{mM}$; addition of hydrogen peroxide to a final concentration of $1 \%$. In each case, cells were harvested $15 \mathrm{~min}$ after exposure to stress conditions. Additionally, C. glutamicum cells were grown in a fermenter in defined CGXII medium to be able to apply high oxygen stress. Therefore, the dissolved oxygen level was shifted from $30 \%$ to $90 \%$. After 2 hours of $90 \%$ dissolved oxygen cells were harvested.

\section{RNA isolation procedures}

For construction of the primary $5^{\prime}$-end library, total RNA was isolated using the RNeasy mini kit along with an RNase-free DNase set (Qiagen, Hilden, Germany) and a DNase I kit (Roche Diagnostics, Mannheim, Germany) according to Hüser et al. (2003) [99].

For creation of the whole transcriptome library, total RNA was isolated using TRIzol (Life Technologies, Darmstadt, Germany). The cell pellet obtained from $800 \mu \mathrm{l}$ of exponentially grown culture was resuspended in $1 \mathrm{ml}$ TRIzol reagent. Cell disruption and homogenization was performed using the homogenizer Precellys 24 (Bertin Technologies, Montigny-le-Bretonneux, France) at a speed of 6.5 for $20 \mathrm{sec}$ twice. After centrifugation at $16,000 \mathrm{~g}$ for $3 \mathrm{~min}$ at $4^{\circ} \mathrm{C}, 200 \mu \mathrm{l}$ chloroform (Roth, Karlsruhe, Germany) was added to the supernatant and shaken vigorously for $30 \mathrm{sec}$ followed by incubation for $1 \mathrm{~min}$ at room temperature and centrifugation at $16,000 \mathrm{~g}$ for $10 \mathrm{~min}$ at $4^{\circ} \mathrm{C}$. For precipitation, 1 volume of isopropanol (Roth, Karlsruhe, Germany) was added to the aqueous supernatant and shaken vigorously. The sample was then incubated on ice for $10 \mathrm{~min}$ and centrifuged at $16,000 \times \mathrm{g}$ (times gravity) for $15 \mathrm{~min}$ at $4^{\circ} \mathrm{C}$. The RNA pellet was washed two times with $75 \%(\mathrm{v} / \mathrm{v})$ ethanol, air-dried and dissolved in $100 \mu \mathrm{l}$ deionized, RNase-free water. The total RNA isolation using TRIzol reagent was followed by DNase treatment with an RNase-Free DNase Set (Qiagen, Hilden, Germany) and a DNase I kit (Sigma-Aldrich, Hamburg, Germany) according to manufacturer's instructions.

To ensure DNA-free RNA samples, a PCR was performed using oligonucleotides which create two different products of about $150 \mathrm{bp}$ and $500 \mathrm{bp}$. Afterwards, the purified total RNA was quantified with a NanoDrop 1000 spectrometer (Peqlab, Erlangen, Germany) and qualified by Agilent RNA Nano 6000 kit on Agilent 2100 Bioanalyzer (Agilent Technologies, Böblingen, Germany).

\section{The native $5^{\prime}$-end protocol}

To analyze the native transcription start sites of C. glutamicum a native 5 '-end RNAseq protocol was developed. The protocol starts with $10 \mu \mathrm{g}$ column-based isolated total RNA. An essential step for effective transcriptome sequencing is the subsequent depletion of stable RNA (rRNA, tRNA) that can constitute more than 95\% of a bacterial transcriptome. Here, we used the hybridization-based Ribo-Zero rRNA removal kit for Gram-positive bacteria that showed an advantageous depletion in the amount of stable RNA for C. glutamicum. Then, the depleted RNA was fragmented to a size of 200 - 500 nt. Primary transcript ends were enriched using Terminator $5^{\prime}$-phosphate-dependent exonuclease (Epicentre, Madison, WI, U.S.A.). This enzyme recognizes and digests processed, non-primary transcripts that offer a monophosphate at their 5 '-end. Bacterial primary transcripts that possess three phosphates at their $5^{\prime}$-ends are not digested and remain in the solution. To be able to ligate RNA adapters at the $5^{\prime}$-ends, the resulting $5^{\prime}$ triphosphate ends were processed to $5^{\prime}$-monophosphate ends by RNA $5^{\prime}$-polyphosphatase (Epicentre, Madison, WI, U.S.A.). The tagging of the 3 '-end of the RNA fragments and the reverse transcription into cDNA is performed ligation-free with a stem-loop DNA adapter with seven free wobble bases at its $3^{\prime}$-end. The wobble bases hybridize to the 3 '-end of a RNA fragment serving as a primer for the reverse transcriptase. The advantage of the use of a stem-loop DNA adapter lies in the avoidance of ligation of an adapter at the 3 '-end which can be 
inefficient depending on the adapter sequence [100] and length of the RNA [101,102]. Additionally, it was shown previously that the use of stem-loop primers for reverse transcription is better than conventional primers in terms of efficiency and specificity [103]. After reverse transcription of the RNA fragments into cDNA, the cDNA fragments were amplified and are then ready for sequencing. The procedure of the native 5 '-end protocol is depicted in Figure 1b.

The detailed steps of the library preparation are described below.

\section{The whole transcriptome protocol}

As a completion to the native $5^{\prime}$-end protocol a whole transcriptome protocol was also developed that enables the sequencing of all transcripts. The procedure of this protocol is very similar to the procedure of the native 5 '-end protocol. The difference between both protocols is the missing enrichment step for primary transcripts and an additional step before the RNA adapter is ligated, where unphosphorylated $5^{\prime}$-ends were phosphorylated and phosphorylated 3 '-ends were dephosphorylated using a T4 polynucleotide kinase (New England BioLabs, Frankfurt am Main, Germany). The procedure of the whole transcriptome protocol is shown in Figure 1a.

\section{Depletion of ribosomal RNA}

For depletion of ribosomal RNA, the Ribo-Zero rRNA removal kit Gram-positive bacteria (Epicentre, Madison, WI, U.S.A.) and purification of the rRNA-depleted sample by ethanol precipitation was used according to manufacturer's instructions.

The purified RNA was then pooled in equimolar amounts (for primary 5 '-end library derived from two cultivation conditions and for whole transcriptome library derived from nine cultivation conditions as described above) and in total $10 \mu \mathrm{g}$ RNA were taken for depletion of rRNA.

\section{Phenol-Chloroform Isoamyl alcohol extraction}

After enzyme treatments, the RNA was purified by phenol-chloroform isoamyl alcohol, 25:24:1, (PCI) extraction. One volume of PCI was added to the sample and shaken vigorously for $30 \mathrm{sec}$ followed by centrifugation at $16,000 \times \mathrm{g}$ for $15 \mathrm{~min}$ at room temperature. For precipitation, 0.3 volumes of sodium acetate $(3 \mathrm{M}$; pH 5.2), $20 \mu \mathrm{g}$ of glycogen (RNA grade; Thermo Fisher Scientific, Schwerte, Germany) and 2.7 volumes of icecold ethanol ( 99\%) were added to the aqueous supernatant and shaken vigorously. The sample was then incubated at $-20^{\circ} \mathrm{C}$ for at least 2 hours and centrifuged at $16,000 \times \mathrm{g}$ for $20 \mathrm{~min}$ at $4^{\circ} \mathrm{C}$. The RNA pellet was washed two times with $75 \%(\mathrm{v} / \mathrm{v})$ ethanol, air-dried and dissolved in deionized, RNase-free water, the volume depending on the following reaction step.

\section{RNA fragmentation}

For both protocols, the RNA needs to be fragmented to sizes of 200 - $500 \mathrm{nt}$. Therefore, 0.25 volumes of fragmentation buffer $(100 \mathrm{mM}$ potassium acetate and $30 \mathrm{mM}$ magnesium acetate dissolved in $200 \mathrm{mM}$ Tris- $\mathrm{HCl} \mathrm{pH}$ 8.1) were added to the RNA sample, shaken vigorously, and incubated at $94^{\circ} \mathrm{C}$ for $2.5 \mathrm{~min}$. After incubation, the sample was mixed vigorously with one volume of ice-cold fragmentation stop buffer (10 mM Tris, $1 \mathrm{mM}$ EDTA, $\mathrm{pH}$ 8) and incubated on ice for $5 \mathrm{~min}$.

After fragmentation, the RNA sample was precipitated with 0.3 volumes of sodium acetate ( $3 \mathrm{M} ; \mathrm{pH} 5.2), 20 \mu \mathrm{g}$ of glycogen (RNA grade), and 2.7 volumes of ice-cold ethanol $(\sim 99 \%)$ at $-20^{\circ} \mathrm{C}$ for at least 2 hours and centrifuged at $16,000 \times \mathrm{g}$ for $20 \mathrm{~min}$ at $4^{\circ} \mathrm{C}$. The RNA pellet was washed two times with $75 \%(\mathrm{v} / \mathrm{v})$ ethanol, air-dried and dissolved in $40 \mu \mathrm{l}$ of deionized, RNase-free water.

Next, RNA fragments larger than $150 \mathrm{nt}$ are precipitated with $10 \mu \mathrm{l}$ of enrichment solution (25\% PEG 8000 (polyethylene glycol), $2.5 \mathrm{M}$ sodium acetate in RNase-free water) on ice for $30 \mathrm{~min}$ and centrifuged at $16,000 \times \mathrm{g}$ for $20 \mathrm{~min}$ at $4^{\circ} \mathrm{C}$. The RNA pellet was washed two times with $75 \%$ $(\mathrm{v} / \mathrm{v})$ ethanol, air-dried and dissolved in deionized, RNase-free water, the volume depending on the following reaction step.

\section{Terminator 5'-phosphate-dependent exonuclease treatment}

For the primary $5^{\prime}$-end protocol, a Terminator $5^{\prime}$-phosphate-dependent exonuclease (Epicentre, Madison, WI, U.S.A.) treatment was included to digest the nonprimary transcripts. This enzyme treatment was used according to manufacturer's instructions followed by PCI extraction.

\section{5'-end repair}

After fragmentation, it is necessary to repair the $5^{\prime}$-RNA ends before ligating adapters. Therefore, the RNA 5' polyphosphatase (Epicentre, Madison, WI, U.S.A.) was used according to manufacturer's instructions. This enzyme converts 5 '-triphosphorylated and 5 ' -diphosphorylated RNA to $5^{\prime}$-monophosphorylated RNA. After RNA $5^{\prime}$-polyphosphatase treatment the RNA was purified by PCI extraction.

In the case of the whole transcriptome library, the RNA 5'-polyphosphatase treatment was followed by a T4 polynucleotide kinase treatment (New England BioLabs, Frankfurt am Main, Germany) according to manufacturer's instructions to phosphorylate unphosphorylated 5 '-RNA ends. Instead of the T4 polynucleotide kinase reaction buffer, T4 RNA ligase reaction buffer supplemented with 
$1 \mathrm{mM}$ ATP was used. After incubation and heat inactivation of T4 polynucleotide kinase the samples are directly used for RNA adapter ligation.

\section{RNA adapter ligation}

An RNA adapter was ligated to the $5^{\prime}$-RNA ends of the prepared RNA fragments. For the ligation, $100 \mu \mathrm{M}$ of RNA adapter was used with T4 RNA ligase 1 (New England BioLabs, Frankfurt am Main, Germany) according to manufacturer's instructions.

\section{Preparation of the stem-loop DNA adapter}

Before use of the stem-loop DNA adapter in the reverse transcription reaction, the stem-loop structure has to be prepared. Therefore, a $20 \mu \mathrm{l}$ aliquot of stem-loop DNA adapter $(100 \mu \mathrm{M})$ was incubated at $98^{\circ} \mathrm{C}$ for $3 \mathrm{~min}$ and cooled to $25^{\circ} \mathrm{C}$ at a rate of $1^{\circ} \mathrm{C}$ per $10 \mathrm{sec}$ in a Mastercycler pro S (Eppendorf).

\section{Reverse transcription and tagging of the 3 '-end of the cDNA in a single step}

After ligation of the $5^{\prime}$-adapter, the RNA fragments are reverse transcribed to cDNA using the ThermoScript RT-PCR system (Life Technologies, Darmstadt, Germany) according to manufacturer's instructions. As cDNA synthesis primer, $1 \mu \mathrm{l}$ of the prepared stem-loop DNA adapter $(100 \mu \mathrm{M})$ was used for one library. After adding all ingredients the reverse transcription was performed at $16^{\circ} \mathrm{C}$ for $30 \mathrm{~min}$ followed by $50^{\circ} \mathrm{C}$ for 1 hour. After heat inactivation of the reverse transcriptase the sample was used for amplification, without removing the RNA template.

\section{Amplification of the CDNA and purification of cDNA library}

The cDNA was amplified with 18 cycles of PCR. For amplification, phusion high-fidelity DNA polymerase (New England BioLabs, Frankfurt am Main, Germany) with $1.5 \%$ DMSO was used according to manufacturer's instructions. The primer annealing was performed at $60^{\circ} \mathrm{C}$ for $30 \mathrm{sec}$ and the extension at $72^{\circ} \mathrm{C}$ for $15 \mathrm{sec}$. After amplification of the cDNA libraries, samples were purified and size-selected (> $150 \mathrm{bp}$ ) by using the Agencourt AMPure XP system (Beckman Coulter, Krefeld, Germany) according manufacturer's instructions. Afterwards, the purified libraries were quantified and qualified with an Agilent 2100 Bioanalyzer (Agilent Technologies, Böblingen, Germany) using the Agilent High Sensitivity DNA kit.

\section{Sequencing of the cDNA library}

Sequencing of the cDNA libraries was carried out on the Genome Analyzer IIx using TruSeq kits (Illumina, San Diego, CA, U.S.A.). The sample derived from the 5 '-end library was sequenced in single read mode with a read length of 27 bases and the whole transcriptome RNAseq library was paired-end sequenced with a read length of $2 \times 27$ bases. Each sample was sequenced on one lane of a flow cell. Data analysis and base calling were accomplished with the Illumina instrument software. After trimming of one low quality base from the 3'-end of each sequenced read, 26 nt reads were used for further analysis.

The sequencing raw data for both libraries is available from ENA (http://www.ebi.ac.uk/ena/), project ID PRJEB4788.

\section{Bioinformatics data analysis \\ Read mapping and data visualization}

Trimmed reads (26 nt) were mapped to the C. glutamicum ATCC 13032 genome sequence [4] with SARU$M A N$ [104], allowing for up to one error per read. In case of the mapping of reads belonging to the whole transcriptome library, the forward and reverse read, if both present and with a maximum distance of $1 \mathrm{~kb}$, were combined to one read, containing the reference sequence as insert. Paired mappings with a distance $>1 \mathrm{~kb}$ were discarded, and paired reads with either only the forward or only the reverse read mapping were retained as single mapping reads. For the visualization of short read alignments, ReadXplorer (Hilker et al., in preparation) was used.

\section{Genome-update of C. glutamicum ATCC 13032}

Since the publication of the C. glutamicum genome [4,5] in 2003, knowledge on many gene functions was collected in more than 600 publications. This data was used to update a $G e n D B$ database [34] that basically contains re-annotated gene starts, some newly annotated genes taken from recent publications and improved gene prediction, and an improved annotation. Furthermore, four non-coding RNAs, 4.5S RNA, 6C RNA, tmRNA, and M1 RNA, which were identified at positions very similar to the Rfam predictions [50] (Additional file 1: Table S10) were also used to update the C. glutamicum genome. For the analysis of the RNAseq data, especially for the determination of the transcription start sites and 5 '-UTRs, this updated genome was used.

\section{Identification of transcription start sites using primary 5 '-end data}

To automatically and systematically detect TSSs, the mapping data of the library enriched for native 5 '-ends was analyzed. First, for each strand and position of the genome, all mappings starting at the given position were counted. As possible TSS all positions on a strand were taken into account that satisfied the following criteria: for a position $i$, the number of read starts $x_{i}$ on that strand at this position exceeded a background threshold $T$ and the ratio $x_{i} / x_{i-1}$ at this position had to exceed a 
threshold $R$. After manual inspection of TSSs, $T$ was set to 14 and $R$ to 5 as these parameters were found to result in a good signal to noise ratio.

Additionally, these automatically detected TSSs, especially those with a distance larger than 300 nt between TSS and TLS, were checked for false-positives based on manual review. A TSS was assumed to be false-positive if no clear accumulation of read starts is observed at the particular position and the found TSS is detected within an uneven gradient of accumulated reads. This specifically applied to TSSs detected within a coding region and/or with a relatively high amount of accumulated reads $(>100)$, where the above-mentioned parameters are not effective.

\section{Identification of novel transcripts}

To identify so far unknown transcripts, the data from the whole transcriptome was used. First, for each strand and each position, the coverage was calculated by adding up all mappings at this position. Then, for each strand and each position $i$ it is checked whether the coverage $c_{i}$ crosses the background threshold $T$. For each position that satisfies this criterion it is checked whether the possible transcript can be extended downstream, with each subsequent position $x$ satisfying the condition $c_{i+x}>T$. Once a position $x$ with $c_{i+x} \leq T$ is reached, this position is considered to be the end of a novel transcript if the transcript does not overlap a known transcript in sense direction.

Every new detected transcript was checked for its genomic context, i.e. whether it is an antisense transcript located antisense to another transcript, an intergenic transcript in between other transcripts, or an intragenic transcript, whereas the transcript start is mapped within an annotated gene. The end position of automatically detected antisense transcripts with length $>1 \mathrm{~kb}$ was manually determined using the whole transcriptome data. Furthermore, new transcripts that were not automatically detected, but derived from identified TSSs belonging to antisense or intergenic transcripts were also categorized as new regions. The end positions of those transcripts were also manually identified. The ends of intragenic transcripts could not be identified, because the appropriate transcript overlaps with the transcript of the annotated gene.

Sequences of intergenic regions were searched for open reading frames using the web program ORF finder [46] with ATG, GTG, TTG, CTG as start codons, and TAA, TGA, TAG as stop codons. Those sequences where an open reading frame was found were searched for homologous proteins in the NCBI reference proteins database (refseq_protein) for Bacteria and Archaea using the NCBI web services and Blastx (November 2013).

\section{Identification of operon structures}

For the identification of operons, again the data from the whole transcriptome library was used. For each gene it was tested whether it is connected to a possible neighboring gene downstream in sense direction by at least $T$ paired read mappings. All consecutive sets of pairs for which a TSS could be identified for the first gene were considered to constitute a primary operon. Sub operons were then defined by TSSs within the primary operon.

\section{Identification of promoter motifs and ribosome binding sites}

The identification of RBSs was started with computing the frequency of purines ( $G$ and $A$ ) compared to that of pyrimidines ( $\mathrm{T}$ and $\mathrm{C}$ ) of each nucleotide within identified 5 '-UTRs of protein-coding genes. For genes with more than one $5^{\prime}$-UTR, only one $5^{\prime}$-UTR (unique $5^{\prime}$ UTR), but with a minimum length of 20 bases, was used for this analysis. Additionally, all analyzed sequences were trimmed to length of 20 bases. Next, a RBS motif was searched within unique 5 '-UTRs with a minimum length of 14 and a trimmed maximum length of 20 bases.

For the identification of promoter and RBSs motifs the web based software tool Improbizer [43] was used. Default settings were applied for both searches.

Improbizer has identified an extended -10 region (four unpreserved leading bases, a core hexamer, and two or three unpreserved lagging bases, respectively). However, for simplification the identified motif was truncated to the core -10 hexamer and used in this work.

Additionally, identified motifs were described in their conservation by upper or lower case bases. An upper case base is used if it occurs in $\geq 80 \%$ of all cases at a certain position within the motif. A lower case base is used, if it arises in $>40 \%$ but $<80 \%$ of all cases at a certain position within the motif. If a base occurs in $\leq 40 \%$ of all cases at a certain position within the motif, a lower case $\mathrm{n}$ is used.

\section{Additional files}

Additional file 1: Table S1. List of identified TSSs with -10 and -35
promoter motifs for the primary sigma factor A predicted by Improbizer
within 60 bases upstream of the TSSs. Table S2. List of genes with
corrected genes starts by RNAseq data. Table S3. List of protein-coding
genes including length and sequence of 5'-UTRs. Table S4. List of ribosome
binding site motifs within max. 20 nt upstream of the initiation codon in
5'-UTRs. Table S5. List of operons, sub-operons, and monocistronic
transcripts. Table S6. List of yet undescribed intergenic transcripts.
Table S7. List of yet undescribed antisense transcripts. Table S8. List of yet
undescribed intragenic transcripts. Table S9. Comparison of predicted
rho-independent terminator for C. glutamicum and the determined
transcript ends. Table S10. Verification of four ncRNAs predicted by
Rfam database for C. glutamicum.


Additional file 2: Figure S1. TSS annotation on the example of the gene cmt1 (cg0413). Black color denotes cumulated reads derived from the primary transcript ends library. The $y$ - and $x$-axis represent coverage and genome position, respectively. The increase of reads starts is determined at six positions (numbers 1 - 6)

Additional file 3: Figure S2 Examples of secondary structures for Rfam predicted 5'-UTRs in C. glutamicum. Structures were predicted using minimum free energy and the partition function in RNAfold provided by the Vienna RNA web server [51]. The initiation codon is highlighted in green, the possible RBS in red.

\section{Abbreviations}

RNAseq: High-throughput RNA sequencing; TSS(s): Transcription start site(s); TLS: Translational start site(s); UTR: Untranslated region; (q)PCR: Quantitative polymerase chain reaction; RACE: Rapid amplification of CDNA ends; nt: Nucleotide(s); RBS(s): Ribosome binding site(s); bp: Base pair(s); w/ o: Without; CPR: Combined pair of reads; FMN: Flavin mononucleotide; TPP: Thiamine pyrophosphate; SAM: S-adenosylmethionine;

NAD: Nicotinamide adenine dinucleotide; ncRNA(s): Non-coding RNA(s); OD: Optical density; $\times g$ : Times gravity; PCl: Phenol-chloroform isoamyl alcohol; PEG: Polyethylene glycol.

\section{Competing interests}

The authors declare that they have no competing interests.

\section{Authors' contributions}

KPS carried out the substantial development of the RNAseq protocols, the experimental and data analysis part of the study, and drafted the manuscript. AM helped in the development of the RNAseq protocols. CR participated in the design of the study, performed sequencing and the bioinformatical analysis of raw RNAseq data, and enabled an (semi)-automated analysis of the data. JK coordinated the study and finalized the manuscript. All authors read and approved the manuscript.

\section{Acknowledgements}

We thank Andreas Albersmeier for assistance with promoter search and Rolf Hilker and Alexander Goesmann for the supply of the RNAseq transcriptome viewer ReadXplorer (Hilker et al., in preparation). We additionally acknowledge the receipt of a grant from the Federal Ministry of Education and Research (BMBF) for the FlexFit consortium (0315585 J), grants from the Federal State of North Rhine-Westphalia for the Technology Platform PolyOmics (w0805wb005) as well as the support of the publication fee by Deutsche Forschungsgemeinschaft and the Open Access Publication Funds of Bielefeld University.

Received: 16 August 2013 Accepted: 3 December 2013 Published: 17 December 2013

\section{References}

1. Hermann T: Industrial production of amino acids by coryneform bacteria. J Biotechnol 2003, 104:155-172.

2. Gopinath V, Murali A, Dhar KS, Nampoothiri KM: Corynebacterium glutamicum as a potent biocatalyst for the bioconversion of pentose sugars to value-added products. Appl Microbiol Biotechnol 2012, 93:95-106.

3. Wendisch VF, Bott M, Kalinowski J, Oldiges M, Wiechert W: Emerging Corynebacterium glutamicum systems biology. J Biotechnol 2006, 124:74-92.

4. Kalinowski J, Bathe B, Bartels D, Bischoff N, Bott M, Burkovski A, Dusch N, Eggeling L, Eikmanns BJ, Gaigalat L, Goesmann A, Hartmann M, Huthmacher K, Krämer R, Linke B, McHardy AC, Meyer F, Möckel B, Pfefferle W, Pühler A, Rey DA, Rückert C, Rupp O, Sahm H, Wendisch VF, Wiegräbe I, Tauch A: The complete Corynebacterium glutamicum ATCC 13032 genome sequence and its impact on the production of L-aspartate-derived amino acids and vitamins. J Biotechnol 2003, 104:5-25.

5. Ikeda M, Nakagawa S: The Corynebacterium glutamicum genome: features and impacts on biotechnological processes. Appl Microbiol Biotechnol 2003, 62:99-109.

6. Brinkrolf $K$, Brune I, Tauch A: The transcriptional regulatory network of the amino acid producer Corynebacterium glutamicum. J Biotechnol 2007, 129:191-211.
7. Pauling J, Röttger R, Tauch A, Azevedo V, Baumbach J: CoryneRegNet 6.0-Updated database content, new analysis methods and novel features focusing on community demands. Nucleic Acids Res 2012, 40:D610-D614.

8. Pátek M, Nešvera J: Sigma factors and promoters in Corynebacterium glutamicum. J Biotechnol 2011, 154:101-113.

9. Mentz A, Neshat A, Pfeifer-Sancar K, Pühler A, Rückert C, Kalinowski J: Comprehensive discovery and characterization of small RNAs in Corynebacterium glutamicum ATCC 13032. BMC Genomics 2013, 14:714.

10. Siezen RJ, Wilson G, Todt T: Prokaryotic whole-transcriptome analysis: deep sequencing and tiling arrays. J Microbial Biotechnol 2010, 3:125-130.

11. Pinto AC, Melo-Barbosa HP, Miyoshi A, Silva A, Azevedo V: Application of RNA-seq to reveal the transcript profile in bacteria. Genet Mol Res 2011, 10:1707-1718

12. Wang Z, Gerstein M, Snyder M: RNA-Seq: a revolutionary tool for transcriptomics. Nat Rev Genet 2009, 10:57-63.

13. Güell M, Yus E, Lluch-Senar M, Serrano L: Bacterial transcriptomics: what is beyond the RNA horiz-ome? Nat Rev Microbiol 2011, 9:658-669.

14. Perkins TT, Kingsley RA, Fookes MC, Gardner PP, James KD, Yu L, Assefa SA, He M, Croucher NJ, Pickard DJ, Maskell DJ, Parkhill J, Choudhary J, Thomson NR, Dougan G: A strand-specific RNA-Seq analysis of the transcriptome of the typhoid bacillus Salmonella typhi. PLoS Genet 2009, 5:e1000569.

15. Sharma CM, Hoffmann S, Darfeuille F, Reignier J, Findeiss S, Sittka A, Chabas S, Reiche K, Hackermüller J, Reinhardt R, Stadler PF, Vogel J: The primary transcriptome of the major human pathogen Helicobacter pylori. Nature 2010, 464:250-255.

16. Chaudhuri RR, Yu L, Kanji A, Perkins TT, Gardner PP, Choudhary J, Maskell DJ, Grant AJ: Quantitative RNA-seq analysis of the Campylobacter jejuni transcriptome. Microbiol (Reading, Engl) 2011, 157:2922-2932.

17. Høvik H, Yu W-H, Olsen I, Chen T: Comprehensive transcriptome analysis of the periodontopathogenic bacterium Porphyromonas gingivalis W83. J Bacteriol 2012, 194:100-114.

18. Nagalakshmi U, Wang Z, Waern K, Shou C, Raha D, Gerstein M, Snyder M: The transcriptional landscape of the yeast genome defined by RNA sequencing. Science 2008, 320:1344-1349.

19. Lister R, O'Malley RC, Tonti-Filippini J, Gregory BD, Berry CC, Millar AH, Ecker JR: Highly integrated single-base resolution maps of the epigenome in Arabidopsis. Cell 2008, 133:523-536.

20. Yang D, Fu Y, Wu X, Xie Y, Nie H, Chen L, Nong X, Gu X, Wang S, Peng X, Yan N, Zhang R, Zheng W, Yang G: Annotation of the transcriptome from Taenia pisiformis and its comparative analysis with three Taeniidae species. PLOS ONE 2012, 7:e32283.

21. Güell M, van Noort V, Yus E, Chen W-H, Leigh-Bell J, Michalodimitrakis K, Yamada T, Arumugam M, Doerks T, Kühner S, Rode M, Suyama M, Schmidt S, Gavin A-C, Bork P, Serrano L: Transcriptome complexity in a genomereduced bacterium. Science 2009, 326:1268-1271.

22. Sorek $R$, Cossart P: Prokaryotic transcriptomics: a new view on regulation, physiology and pathogenicity. Nat Rev Genet 2010, 11:9-16.

23. Dornenburg JE, Devita AM, Palumbo MJ, Wade JT: Widespread antisense transcription in Escherichia coli. MBio 2010, 1:e00024-10.

24. Raghavan R, Groisman EA, Ochman H: Genome-wide detection of novel regulatory RNAs in E. coli. Genome Res 2011, 21:1487-1497.

25. Denoeud F, Aury J-M, Da Silva C, Noel B, Rogier O, Delledonne M, Morgante M, Valle G, Wincker P, Scarpelli C, Jaillon O, Artiguenave F: Annotating genomes with massive-scale RNA sequencing. Genome Biol 2008, 9:R175.

26. Frank S, Klockgether J, Hagendorf P, Geffers R, Schöck U, Pohl T, Davenport CF, Tümmler B: Pseudomonas putida KT2440 genome update by CDNA sequencing and microarray transcriptomics. Environ Microbiol 2011 13:1309-1326.

27. Jacob F, Monod J: On the Regulation of Gene Activity. Cold Spring Harb Symp Quant Biol 1961, 26:193-211.

28. Shine J, Dalgarno L: Determinant of cistron specificity in bacterial ribosomes. Nature 1975, 254:34-38.

29. Lawrence JG, Roth JR: Selfish operons: horizontal transfer may drive the evolution of gene clusters. Genetics 1996, 143:1843-1860.

30. Osbourn AE, Field B: Operons. Cell Mol Life Sci 2009, 66:3755-3775.

31. Cloonan N, Forrest ARR, Kolle G, Gardiner BBA, Faulkner GJ, Brown MK, Taylor DF, Steptoe AL, Wani S, Bethel G, Robertson AJ, Perkins AC, Bruce SJ, Lee CC, Ranade SS, Peckham HE, Manning JM, McKernan KJ, Grimmond SM: Stem cell transcriptome profiling via massive-scale mRNA sequencing. Nat Methods 2008, 5:613-619. 
32. Vockenhuber M-P, Sharma CM, Statt MG, Schmidt D, Xu Z, Dietrich S, Liesegang $H$, Mathews DH, Suess B: Deep sequencing-based identification of small non-coding RNAs in Streptomyces coelicolor. RNA Biol 2011, 8:468-477.

33. Passalacqua KD, Varadarajan A, Ondov BD, Okou DT, Zwick ME, Bergman $\mathrm{NH}$ : Structure and complexity of a bacterial transcriptome. J Bacteriol 2009, 191:3203-3211.

34. Meyer F, Goesmann A, McHardy AC, Bartels D, Bekel T, Clausen J, Kalinowski J, Linke B, Rupp O, Giegerich R, Pühler A: GenDB-an open source genome annotation system for prokaryote genomes. Nucleic Acids Res 2003, 31:2187-2195.

35. Jacob F: Genetics of the bacterial cell. Science 1966, 152:1470-1478.

36. Szybalski W, Kubinski H, Sheldrick P: Pyrimidine clusters on the transcribing strand of DNA and their possible role in the initiation of RNA synthesis. Cold Spring Harb Symp Quant Biol 1966, 31:123-127.

37. Ishihama A: Promoter selectivity of prokaryotic RNA polymerases. Trends Genet 1988, 4:282-286.

38. Ishihama A: Functional modulation of Escherichia coli RNA polymerase. Annu Rev Microbiol 2000, 54:499-518.

39. Paget MSB, Helmann JD: The sigma70 family of sigma factors. Genome Biol 2003, 4:203.

40. Pribnow D: Nucleotide sequence of an RNA polymerase binding site at an early T7 promoter. Proc Natl Acad Sci USA 1975, 72:784-788.

41. Seeburg PH, Nüsslein C, Schaller H: Interaction of RNA polymerase with promoters from bacteriophage fd. Eur J Biochem 1977, 74:107-113.

42. Hawley DK, McClure WR: Compilation and analysis of Escherichia coli promoter DNA sequences. Nucleic Acids Res 1983, 11:2237-2255.

43. Ao W, Gaudet J, Kent WJ, Muttumu S, Mango SE: Environmentally induced foregut remodeling by PHA-4/FoxA and DAF-12/NHR. Science 2004, 305:1743-1746.

44. Pátek M, Nesvera J, Guyonvarch A, Reyes O, Leblon G: Promoters of Corynebacterium glutamicum. J Biotechnol 2003, 104:311-323.

45. Crooks GE, Hon G, Chandonia J-M, Brenner SE: WebLogo: a sequence logo generator. Genome Res 2004, 14:1188-1190.

46. ORF finder. http://www.gen-info.osaka-u.ac.jp/ uhmin/study/orf/index.cgi.

47. Powell S, Szklarczyk D, Trachana K, Roth A, Kuhn M, Muller J, Arnold R, Rattei T, Letunic I, Doerks T, Jensen $L$, von Mering C, Bork P: eggNOG v3.0: orthologous groups covering 1133 organisms at 41 different taxonomic ranges. Nucleic Acids Res 2012, 40:D284-D289.

48. Winkler WC, Breaker RR: Regulation of bacterial gene expression by riboswitches. Annu Rev Microbiol 2005, 59:487-517.

49. Fröhlich KS, Vogel J: Activation of gene expression by small RNA. Curr Opin Microbiol 2009, 12:674-682.

50. Gardner PP, Daub J, Tate J, Moore BL, Osuch IH, Griffiths-Jones S, Finn RD, Nawrocki EP, Kolbe DL, Eddy SR, Bateman A: Rfam: Wikipedia, clans and the "decimal" release. Nucleic Acids Res 2011, 39:D141-D145.

51. Gruber AR, Lorenz R, Bernhart SH, Neuböck R, Hofacker IL: The Vienna RNA websuite. Nucleic Acids Res 2008, 36:W70-W74.

52. Mandal M, Breaker RR: Gene regulation by riboswitches. Nat Rev Mol Cell Biol 2004, 5:451-463

53. Nudler E, Mironov AS: The riboswitch control of bacterial metabolism. Trends Biochem Sci 2004, 29:11-17.

54. Kingsford CL, Ayanbule K, Salzberg SL: Rapid, accurate, computational discovery of Rho-independent transcription terminators illuminates their relationship to DNA uptake. Genome Biol 2007, 8:R22.

55. Hillier LW, Reinke V, Green P, Hirst M, Marra MA, Waterston RH: Massively parallel sequencing of the polyadenylated transcriptome of $C$. elegans. Genome Res 2009, 19:657-666.

56. Oliver HF, Orsi RH, Ponnala L, Keich U, Wang W, Sun Q, Cartinhour SW, Filiatrault MJ, Wiedmann M, Boor KJ: Deep RNA sequencing of $L$. monocytogenes reveals overlapping and extensive stationary phase and sigma B-dependent transcriptomes, including multiple highly transcribed noncoding RNAs. BMC Genomics 2009, 10:641.

57. Jacob NM, Kantardjieff A, Yusufi FNK, Retzel EF, Mulukutla BC, Chuah SH, Yap M, Hu W-S: Reaching the depth of the Chinese hamster ovary cell transcriptome. Biotechnol Bioeng 2010, 105:1002-1009.

58. Filichkin SA, Priest HD, Givan SA, Shen R, Bryant DW, Fox SE, Wong W-K, Mockler TC: Genome-wide mapping of alternative splicing in Arabidopsis thaliana. Genome Res 2010, 20:45-58.

59. Keilty S, Rosenberg M: Constitutive function of a positively regulated promoter reveals new sequences essential for activity. J Biol Chem 1987, 262:6389-6395.
60. Wösten MM: Eubacterial sigma-factors. FEMS Microbiol Rev 1998, 22:127-150

61. Schibler U, Sierra F: Alternative promoters in developmental gene expression. Annu Rev Genet 1987, 21:237-257.

62. Winkler W, Nahvi A, Breaker RR: Thiamine derivatives bind messenger RNAs directly to regulate bacterial gene expression. Nature 2002, 419:952-956.

63. Vitreschak AG, Rodionov DA, Mironov AA, Gelfand MS: Riboswitches: the oldest mechanism for the regulation of gene expression? Trends Genet 2004, 20:44-50.

64. Giuliodori AM, Di Pietro F, Marzi S, Masquida B, Wagner R, Romby P, Gualerzi CO, Pon CL: The cspA mRNA is a thermosensor that modulates translation of the cold-shock protein CspA. Mol Cell 2010, 37:21-33.

65. Kulis-Horn RK, Persicke M, Kalinowski J: Histidine biosynthesis, its regulation and biotechnological application in Corynebacterium glutamicum. Microb Biotechnol 2014, 7(1):5-25.

66. Seliverstov AV, Putzer H, Gelfand MS, Lyubetsky VA: Comparative analysis of RNA regulatory elements of amino acid metabolism genes in Actinobacteria. BMC Microbiol 2005, 5:54.

67. Michalodimitrakis K, Isalan M: Engineering prokaryotic gene circuits. FEMS Microbiol Rev 2009, 33:27-37.

68. Emory SA, Bouvet P, Belasco JG: A 5'-terminal stem-loop structure can stabilize mRNA in Escherichia coli. Genes Dev 1992, 6:135-148.

69. Belasco JG: All things must pass: contrasts and commonalities in eukaryotic and bacterial mRNA decay. Nat Rev Mol Cell Biol 2010, $11: 467-478$

70. Moll I, Grill S, Gualerzi CO, Bläsi U: Leaderless mRNAs in bacteria: surprises in ribosomal recruitment and translational control. Mol Microbiol 2002, 43:239-246.

71. Wurtzel O, Sapra R, Chen F, Zhu Y, Simmons BA, Sorek R: A single-base resolution map of an archaeal transcriptome. Genome Res 2010, 20:133-141.

72. Kixmüller D, Strahl H, Wende A, Greie J-C: Archaeal transcriptional regulation of the prokaryotic $\mathrm{KdpFABC}$ complex mediating $\mathrm{K}(+)$ uptake in H. salinarum. Extremophiles 2011, 15:643-652.

73. Jäger D, Sharma CM, Thomsen J, Ehlers C, Vogel J, Schmitz RA: Deep sequencing analysis of the Methanosarcina mazei Gö1 transcriptome in response to nitrogen availability. PNAS 2009, 106:21878-21882.

74. Langer D, Hain J, Thuriaux P, Zillig W: Transcription in archaea: similarity to that in eucarya. Proc Natl Acad Sci USA 1995, 92:5768-5772.

75. Brock JE, Pourshahian S, Giliberti J, Limbach PA, Janssen GR: Ribosomes bind leaderless mRNA in Escherichia coli through recognition of their 5'-terminal AUG. RNA 2008, 14:2159-2169.

76. Moll I, Hirokawa G, Kiel MC, Kaji A, Bläsi U: Translation initiation with 70S ribosomes: an alternative pathway for leaderless mRNAs. Nucleic Acids Res 2004, 32:3354-3363.

77. Malys N, McCarthy JEG: Translation initiation: variations in the mechanism can be anticipated. Cell Mol Life Sci 2011, 68:991-1003.

78. Shine J, Dalgarno L: The 3'-terminal sequence of Escherichia coli $16 \mathrm{~S}$ ribosomal RNA: complementarity to nonsense triplets and ribosome binding sites. Proc Natl Acad Sci USA 1974, 71:1342-1346.

79. Ringquist S, Shinedling S, Barrick D, Green L, Binkley J, Stormo GD, Gold L: Translation initiation in Escherichia coli: sequences within the ribosome-binding site. Mol Microbiol 1992, 6:1219-1229.

80. McCarthy JE, Brimacombe R: Prokaryotic translation: the interactive pathway leading to initiation. Trends Genet 1994, 10:402-407.

81. Chapon C: Expression of malT, the regulator gene of the maltose region in Escherichia coli, is limited both at transcription and translation. EMBO J 1982, 1:369-374.

82. Ozbudak EM, Thattai M, Kurtser I, Grossman AD, van Oudenaarden A: Regulation of noise in the expression of a single gene. Nat Genet 2002, 31:69-73.

83. Gold L: Posttranscriptional regulatory mechanisms in Escherichia coli. Annu Rev Biochem 1988, 57:199-233.

84. Stormo GD, Schneider TD, Gold LM: Characterization of translational initiation sites in E. coli. Nucleic Acids Res 1982, 10:2971-2996.

85. Jacob F, Monod J: Genetic regulatory mechanisms in the synthesis of proteins. J Mol Biol 1961, 3:318-356.

86. Qiu Y, Cho B-K, Park YS, Lovley D, Palsson B $\varnothing$, Zengler K: Structural and operational complexity of the Geobacter sulfurreducens genome. Genome Res 2010, 20:1304-1311. 
87. Koide T, Reiss DJ, Bare JC, Pang WL, Facciotti MT, Schmid AK, Pan M, Marzolf B, Van PT, Lo F-Y, Pratap A, Deutsch EW, Peterson A, Martin D, Baliga NS: Prevalence of transcription promoters within archaeal operons and coding sequences. Mol Syst Biol 2009, 5:285.

88. Löchelt M, Muranyi W, Flügel RM: Human foamy virus genome possesses an internal, Bel-1-dependent and functional promoter. Proc Natl Acad Sci USA 1993, 90:7317-7321.

89. Schoenfeld A, Davidowitz EJ, Burk RD: A second major native von HippelLindau gene product, initiated from an internal translation start site, functions as a tumor suppressor. Proc Natl Acad Sci USA 1998, 95:8817-8822.

90. Denoeud F, Kapranov P, Ucla C, Frankish A, Castelo R, Drenkow J, Lagarde J, Alioto T, Manzano C, Chrast J, Dike S, Wyss C, Henrichsen CN, Holroyd N, Dickson MC, Taylor R, Hance Z, Foissac S, Myers RM, Rogers J, Hubbard T, Harrow J, Guigó R, Gingeras TR, Antonarakis SE, Reymond A: Prominent use of distal $5^{\prime}$ transcription start sites and discovery of a large number of additional exons in ENCODE regions. Genome Res 2007, 17:746-759.

91. Thomason MK, Storz G: Bacterial antisense RNAs: how many are there, and what are they doing? Annu Rev Genet 2010, 44:167-188.

92. Wilson KS, von Hippel PH: Transcription termination at intrinsic terminators: the role of the RNA hairpin. Proc Natl Acad Sci U S A 1995 92:8793-8797.

93. Gusarov I, Nudler E: The Mechanism of Intrinsic Transcription Termination. Molecular Cell 1999, 3:495-504.

94. d'Aubenton CY, Brody E, Thermes C: Prediction of rho-independent Escherichia coli transcription terminators: A statistical analysis of their RNA stem-loop structures. J Mol Biol 1990, 216:835-858.

95. Schmeissner U, McKenney K, Rosenberg M, Court D: Removal of a terminator structure by RNA processing regulates int gene expression. J Mol Biol 1984, 176:39-53.

96. Schroth GP, Ho PS: Occurrence of potential cruciform and H-DNA forming sequences in genomic DNA. Nucleic Acids Res 1995, 23:1977-1983.

97. Ruan X, Ruan Y: Genome Wide Full-Length Transcript Analysis Using 5' and 3' Paired-End-Tag Next Generation Sequencing (RNA-PET). In Transcriptional Regulation. Edited by Vancura A. New York: Springer; 2012:535-562.

98. Keilhauer C, Eggeling L, Sahm H: Isoleucine synthesis in Corynebacterium glutamicum: molecular analysis of the ilvB-ilvN-ilvC operon. J Bacteriol 1993, 175:5595-5603.

99. Hüser AT, Becker A, Brune I, Dondrup M, Kalinowski J, Plassmeier J, Pühler A, Wiegräbe I, Tauch A: Development of a Corynebacterium glutamicum DNA microarray and validation by genome-wide expression profiling during growth with propionate as carbon source. J Biotechnol 2003, 106:269-286.

100. Romaniuk E, McLaughlin LW, Neilson T, Romaniuk PJ: The effect of acceptor oligoribonucleotide sequence on the T4 RNA ligase reaction. Eur J Biochem 1982, 125:639-643.

101. Xu J, Lapham J, Crothers DM: Determining RNA solution structure by segmental isotopic labeling and NMR: application to Caenorhabditis elegans spliced leader RNA 1. Proc Natl Acad Sci USA 1996, 93:44-48.

102. Nishigaki K, Taguchi K, Kinoshita Y, Aita T, Husimi Y: Y-ligation: an efficient method for ligating single-stranded DNAs and RNAs with T4 RNA ligase. Mol Divers 1998, 4:187-190.

103. Chen C, Ridzon DA, Broomer AJ, Zhou Z, Lee DH, Nguyen JT, Barbisin M, Xu NL, Mahuvakar VR, Andersen MR, Lao KQ, Livak KJ, Guegler KJ: Real-time quantification of microRNAs by stem-loop RT-PCR. Nucleic Acids Res 2005, 33:e179.

104. Blom J, Jakobi T, Doppmeier D, Jaenicke S, Kalinowski J, Stoye J, Goesmann A Exact and complete short-read alignment to microbial genomes using Graphics Processing Unit programming. Bioinformatics 2011, 27:1351-1358.

doi:10.1186/1471-2164-14-888

Cite this article as: Pfeifer-Sancar et al:: Comprehensive analysis of the Corynebacterium glutamicum transcriptome using an improved RNAseq technique. BMC Genomics 2013 14:888.

\section{Submit your next manuscript to BioMed Central and take full advantage of:}

- Convenient online submission

- Thorough peer review

- No space constraints or color figure charges

- Immediate publication on acceptance

- Inclusion in PubMed, CAS, Scopus and Google Scholar

- Research which is freely available for redistribution

Submit your manuscript at www.biomedcentral.com/submit 